\title{
Green's Functions for Heat Conduction for Unbounded and Bounded Rectangular Spaces: Time and Frequency Domain Solutions
}

\author{
Inês Simões, ${ }^{1,2}$ António Tadeu, ${ }^{1,2}$ and Nuno Simões ${ }^{1,2}$ \\ ${ }^{1}$ ITeCons, Rua Pedro Hispano, s/n, 3030-289 Coimbra, Portugal \\ ${ }^{2}$ Department of Civil Engineering, University of Coimbra, Rua Luís Reis Santos, Pólo II, 3030-788 Coimbra, Portugal \\ Correspondence should be addressed to Nuno Simões; nasimoes@dec.uc.pt
}

Received 11 September 2015; Accepted 19 October 2015

Academic Editor: Assunta Andreozzi

Copyright (C) 2016 Inês Simões et al. This is an open access article distributed under the Creative Commons Attribution License, which permits unrestricted use, distribution, and reproduction in any medium, provided the original work is properly cited.

\begin{abstract}
This paper presents a set of fully analytical solutions, together with explicit expressions, in the time and frequency domain for the heat conduction response of homogeneous unbounded and of bounded rectangular spaces (three-, two-, and one-dimensional spaces) subjected to point, line, and plane heat diffusion sources. Particular attention is given to the case of spatially sinusoidal, harmonic line sources. In the literature this problem is often referred to as the two-and-a-half-dimensional fundamental solution or 2.5D Green's functions. These equations are very useful for formulating three-dimensional thermodynamic problems by means of integral transforms methods and/or boundary elements. The image source technique is used to build up different geometries such as half-spaces, corners, rectangular pipes, and parallelepiped boxes. The final expressions are verified here by applying the equations to problems for which the solution is known analytically in the time domain.
\end{abstract}

\section{Introduction}

Problems in thermodynamics can often be solved with the aid of formulas or expressions known as Green's functions. These functions, or fundamental solutions, relate the field variables (heat fluxes and temperatures) at some location in a solid body caused by thermodynamic sources placed elsewhere in the medium.

The fundamental solutions most often used are point sources in a three-dimensional (3D), infinite homogeneous space; line sources acting within two-dimensional (2D) spaces; and plane sources heating one-dimensional (1D) spaces. The reason for these choices is that these three fundamental solutions are known in closed-form in time domain and have a relatively simple structure [1].

They are frequently combined to simulate heat conduction in the time domain or in a transform space defined by the Laplace transform, in half-spaces, infinite plates, rectangular $2 \mathrm{D}$ spaces, wedges, and rectangular 3D spaces [1-3]. Solutions have also been proposed to deal with multilayer systems, and they include the matrix method [1], the thermal quadrupole method [3], the thin layer method [4], and methods based on the definition of potentials [5-7]. Chen et al. have described the use of image method to solve $2 \mathrm{D}$ and $3 \mathrm{D}$ problems in unbounded and half-space domains containing circular or spherical shaped boundaries [8-10].

This paper compiles alternative fundamental solutions in explicit form, specifically Green's functions for harmonic 2D and 3D and harmonic (steady state) line sources whose amplitude varies sinusoidally in the third dimension. This last solution, which is often referred to in the literature as the $2.5 \mathrm{D}$ problem, can be of significant value when formulating $3 \mathrm{D}$ thermodynamics problems via boundary elements together with integral transforms. In addition, the proposed Green's functions are combined using an image source technique to model a half-space, a corner, a layer system, a laterally confined layer system, a solid rectangular column, a solid rectangular column with an end cross section, and a 3D parallelepiped inclusion. To the best of our knowledge, this is the first such derivation that promises to be efficient for formulating $3 \mathrm{D}$ thermodynamics problems using boundary elements and integral transforms. 
Time domain solutions are obtained by applying inverse Fourier transforms, using complex frequencies to avoid aliasing phenomena. These solutions are validated by comparing computed responses with those obtained directly in the time domain.

\section{Fundamental Solution}

The transient heat transfer by conduction in an infinite, homogeneous space can be described by the diffusion equation in Cartesian coordinates:

$$
\left(\frac{\partial^{2}}{\partial x^{2}}+\frac{\partial^{2}}{\partial y^{2}}+\frac{\partial^{2}}{\partial z^{2}}\right) T=\frac{1}{K} \frac{\partial T}{\partial t},
$$

in which $t$ is time, $T(x, y, z, t)$ is the temperature at a point $(x, y, z)$ in the domain, and $K$ is the thermal diffusivity defined by $\lambda /(\rho c)$, where $\lambda$ is the thermal conductivity, $\rho$ is the density, and $c$ is the specific heat of medium.

The solution of (1) can be obtained in the frequency domain after the application of a Fourier transform in the time domain, which leads to the following equation:

$$
\left(\nabla^{2}+\left(\sqrt{\frac{-i \omega}{K}}\right)^{2}\right) T(x, y, z, \omega)=0
$$

where $i=\sqrt{-1}, k_{1}=\sqrt{-i \omega / K}$ and $\omega$ is the frequency.

Consider first an infinite, homogeneous space subjected at $\left(x_{0}, y_{0}, z_{0}\right)$ to a harmonic point heat source of the form $\delta\left(x-x_{0}\right) \delta\left(y-y_{0}\right) \delta\left(z-z_{0}\right) e^{i \omega t}$. In this expression, $\delta\left(x-x_{0}\right)$, $\delta\left(y-y_{0}\right)$, and $\delta\left(z-z_{0}\right)$ are Dirac delta functions, and $\omega$ is the frequency of the source. The response of this heat source can be expressed by

$$
\widehat{T}_{f}(x, y, z, \omega)=\frac{e^{-\sqrt{i \omega / K} \sqrt{r^{2}+\left(z-z_{0}\right)^{2}}}}{2 \lambda \sqrt{r^{2}+\left(z-z_{0}\right)^{2}}},
$$

where $r=\sqrt{\left(x-x_{0}\right)^{2}+\left(y-y_{0}\right)^{2}}$.

Consider next an infinite, homogeneous space subjected to a spatially varying line heat source of the form $\delta(x-$ $\left.x_{0}\right) \delta\left(y-y_{0}\right) e^{i\left(\omega t-k_{z} z\right)}$, with $k_{z}$ being the wavenumber in $z$. This source acts in one of the three coordinate directions, passes through $\left(x_{0}, y_{0}\right)$, and varies sinusoidally in the $z$ (i.e., third) dimension. This type of source is often referred to in the literature as a $2.5 \mathrm{D}$ source. The response to this source can be obtained by applying a spatial Fourier transform in the $z$ direction to the equations for a point heat load.

Applying a Fourier transformation in the $z$ direction leads to the solution

$$
\widetilde{T}_{f}\left(x, y, k_{z}, \omega\right)=\frac{-i}{4 \lambda} H_{0}\left(k_{1 z} r\right)
$$

where $k_{1 z}=\sqrt{-i \omega / K-k_{z}^{2}}, H_{0}()$ are Hankel functions of the second kind and order 0 .

The full 3D solution can then be achieved by applying an inverse Fourier transform in the $k_{z}$ domain. This inverse Fourier transformation can be expressed as a discrete summation if we assume the existence of virtual sources, equally spaced at $L_{z}$ along $z$, which enables the solution to be obtained by solving a limited number of $2 \mathrm{D}$ problems,

$$
\begin{aligned}
& \widehat{T}_{f}(x, y, z, \omega) \\
& =\frac{2 \pi}{L_{z}} \frac{-i}{4 \lambda} \sum_{m_{k}=-M}^{M} H_{0}\left(\sqrt{-\frac{i \omega}{K}-k_{z m}^{2} r}\right) e^{-i k_{z m} z}
\end{aligned}
$$

with $k_{z m}$ being the axial wavenumber given by $k_{z m}=$ $\left(2 \pi / L_{z}\right) m_{k}$. The distance $L_{z}$ chosen must be big enough to prevent spatial contamination from the virtual sources.

Equation (4) can be further manipulated and written as a continuous superposition of heat plane phenomena,

$$
\begin{aligned}
& \widetilde{T}_{f}\left(x, y, k_{z}, \omega\right) \\
& \quad=\frac{-i}{4 \pi \lambda} \int_{-\infty}^{+\infty}\left(\frac{e^{-i k_{1 z x}\left|y-y_{0}\right|}}{k_{1 z x}}\right) e^{-i k_{x}\left(x-x_{0}\right)} d k_{x}
\end{aligned}
$$

where $k_{1 z x}=\sqrt{-i \omega / K-k_{z}^{2}-k_{x}^{2}}$ and $\operatorname{Im}\left(k_{1 z x}\right) \leq 0$, and the integration is performed with respect to the horizontal wave number $\left(k_{x}\right)$ in the $x$ direction.

Assuming the existence of an infinite number of virtual sources, we can discretize these continuous integrals. The integral in the above equation can be transformed into a summation if an infinite number of such sources are distributed along the $x$ direction, spaced at equal intervals $L_{x}$. The above equation can then be written as

$$
\widetilde{T}_{f}\left(x, y, k_{z}, \omega\right)=E_{0} \sum_{n_{x}=-\infty}^{n=+\infty}\left(\frac{E}{k_{1 z n}}\right) E_{d},
$$

where $E_{0}=-i /\left(2 \lambda L_{x}\right), E=e^{-i k_{1 z n}|y|}, E_{d}=e^{-i k_{n}(x)}, k_{1 z n}=$ $\sqrt{-i \omega / K-k_{z}^{2}-k_{n}^{2}}$ and $\operatorname{Im}\left(k_{1 z n}\right) \leq 0$, and $k_{n}=\left(2 \pi / L_{x}\right) n_{x}$, which can in turn be approximated by a finite sum of equations $(N)$. Note that $k_{z}=0$ is the $2 \mathrm{D}$ case, $\widetilde{T}_{f}(x, y, \omega)=$ $E_{0} \sum_{n_{x}=-\infty}^{n=+\infty}\left(E / k_{1 n}\right) E_{d}$ with $k_{1 n}=\sqrt{-i \omega / K-k_{n}^{2}}$.

Next, the above Green's functions are combined so as to define Green's functions for a half-space, a corner, a single layer system, a $U$ system, a solid rectangular pipe, a solid open box, and a 3D parallelepiped box. Expressions in frequency and time solutions are provided. The time solutions obtained after the application of inverse spatial and frequency Fourier transforms are compared with those given by Green's functions defined directly in the time domain.

Green's functions for the different spaces are determined using the image source method. By this method a distribution of virtual sources and sinks are combined so as to give null temperatures (Dirichlet boundary conditions) or heat fluxes on the required boundaries (Neumann boundary conditions). Other boundary conditions, such as Robin, are not studied in this paper. In the case of solid bodies bounded by two parallel surfaces the number of sources, placed perpendicular to the surfaces, is theoretically infinite. 
TABLE 1

\begin{tabular}{lcr} 
& $r_{i j k}^{l m n}=\sqrt{\left(\bar{x}_{i}\right)^{2}+\left(\bar{y}_{j}\right)^{2}+\left(\bar{z}_{k}\right)^{2}}$ & \\
\hline $\bar{x}_{0}=x-x_{0}$ & $\bar{y}_{0}=y-y_{0}$ & $\bar{z}_{0}=z-z_{0}$ \\
\hline $\bar{x}_{1}=x+x_{0}-2 d_{1} l$ & $\bar{y}_{1}=y+y_{0}-2 d_{2} m$ & $\bar{z}_{1}=z+z_{0}-2 d_{3} n$ \\
\hline $\bar{x}_{2}=x+x_{0}+2 d_{1}(l-1)$ & $\bar{y}_{2}=y+y_{0}+2 d_{2}(m-1)$ & $\bar{z}_{2}=z+z_{0}+2 d_{3}(n-1)$ \\
\hline $\bar{x}_{3}=x-x_{0}-2 d_{1} l$ & $\bar{y}_{3}=y-y_{0}-2 d_{2} m$ & $\bar{z}_{3}=z-z_{0}-2 d_{3} n$ \\
\hline $\bar{x}_{4}=x-x_{0}+2 d_{1} l$ & $\bar{y}_{4}=y-y_{0}+2 d_{2} m$ & $\bar{z}_{4}=z-z_{0}+2 d_{3} n$ \\
\hline
\end{tabular}

The superscripts $l, m$, and $n$ identify the position of the virtual sources along the $x, y$, and $z$ directions, respectively. The upper value of $l, m$, and $n$ is defined by the convergence criteria. Each value of $l, m$, and $n$ is associated with four possible source positions, which are identified by the subscripts $i, j$, and $k$ for the $x, y$, and $z$ directions, respectively. Thus, $i, j$, and $k$ may take the values of $1,2,3$, and 4 .

The use of complex frequencies allows the contribution of the sources placed at greater distances to vanish and so to limit the number of the virtual sources. The use of complex frequencies with a small imaginary part, taking the form $\omega_{c}=$ $\omega-i \eta$ (where $\omega=0.7 \Delta \omega$ and $\Delta \omega$ is the frequency increment), has the additional effect of avoiding the aliasing phenomena. This shift in the frequency domain is subsequently taken into account in the time domain by means of an exponential window, $e^{\eta t}$, applied to the response.

Green's functions are validated assuming that the medium is subject to a Dirac delta source. This type of source would require the solution to be computed in the frequency domain $[0.0, \infty] \mathrm{Hz}$. However, the response does not need to be computed for a very large number of frequencies since it decays very quickly as the frequency decays. Note that the static response for the frequency $0.0 \mathrm{~Hz}$ can be calculated thanks to the use of complex frequencies.

The number of virtual sources used depends directly on the predefined convergence criterion. As we move from one dimension to two dimensions and then to three dimensions, the number of sources grows significantly. Thus, although the method converges rapidly, the cost of computation grows significantly as we move from a one-dimensional to a threedimensional problem.

\section{Green's Functions}

Green's functions in the time and frequency domain will be grouped for the following three cases:

(i) unbounded space, which includes Green's functions for $1 \mathrm{D}, 2 \mathrm{D}$, and $3 \mathrm{D}$ sources;

(ii) two-dimensional space, which contains Green's functions for a half-space, a space bounded by two perpendicular planes, a single layer system, a U system, and a solid rectangular pipe, when subjected to $2 \mathrm{D}$ and $3 \mathrm{D}$ sources;

(iii) three-dimensional space, which compiles Green's functions for point sources placed in a solid open box and in a $3 \mathrm{D}$ parallelepiped box.

Special attention is given to the $2.5 \mathrm{D}$ solution in all cases since it enables the computation of the $3 \mathrm{D}$ heat field as a summation of 2D sources with varying spatial wavenumbers. Different boundary conditions are assumed and combined, namely, null temperatures or null heat fluxes. For each case, a scheme of the geometry is first illustrated (Figures 9-16) and then Green's functions are presented in the time and frequency domains. To verify the proposed solutions, responses in the time domain are included and computed directly in the time domain and in the frequency domain. A selection of results is presented in Figures 1-8 to illustrate the agreement between different solutions. Each figure includes a legend that indicates the comparisons performed.

All results showed a good agreement between the different formulations for all cases.

In the examples provided the Dirac delta source is positioned at the coordinate $\left(x_{0}=0.2 \mathrm{~m}, y_{0}=0.1 \mathrm{~m}, z_{0}=\right.$ $0.15 \mathrm{~m}$ ). This medium is homogeneous and is characterized by a thermal conductivity of $\lambda=426.0 \mathrm{~W} \cdot \mathrm{m}^{-1} \cdot{ }^{\circ} \mathrm{C}^{-1}$, a density $\rho=10500.0 \mathrm{~kg} \cdot \mathrm{m}^{-3}$, and a specific heat $c=235.0 \mathrm{~J} \cdot \mathrm{kg}^{-1}$. ${ }^{\circ} \mathrm{C}^{-1}$. Computations are performed in the frequency range $[0.0,40.96] \mathrm{Hz}$ with a frequency increment of $0.01 \mathrm{~Hz}$. The heat field is computed at the receiver point $R(x=0.25 \mathrm{~m}, y=$ $0.15 \mathrm{~m}, z=0.05 \mathrm{~m})$. The responses obtained with the time formulation (used to validate the frequency formulation) are determined for a time window $[0.0,100.0] \mathrm{s}$ with a time step of $0.0061 \mathrm{~s}$. Depending on the type of geometry the medium may be bounded by planes placed at $x=0.0 \mathrm{~m}, y=0.0 \mathrm{~m}$, $z=0.0 \mathrm{~m}, x=d_{1}=0.30 \mathrm{~m}, y=d_{2}=0.25 \mathrm{~m}$, or $/$ and $z=d_{3}=0.20 \mathrm{~m}$.

The notation in Table 1 is used.

\subsection{Unbounded Space. See Figure 9.}

Equations for an Unbounded Space, Assuming 1D, 2D, and 3D Heat Sources. 1D source is as follows:

$$
\begin{aligned}
T(y, t) & =\frac{e^{-\left|\bar{y}_{0}\right|^{2} / 4 K \tau}}{\rho c(4 \pi K \tau)^{1 / 2}}, \\
T(y, \omega) & =\frac{-i}{2 \lambda} \frac{e^{-i k_{1}\left|\bar{y}_{0}\right|}}{k_{1}} .
\end{aligned}
$$




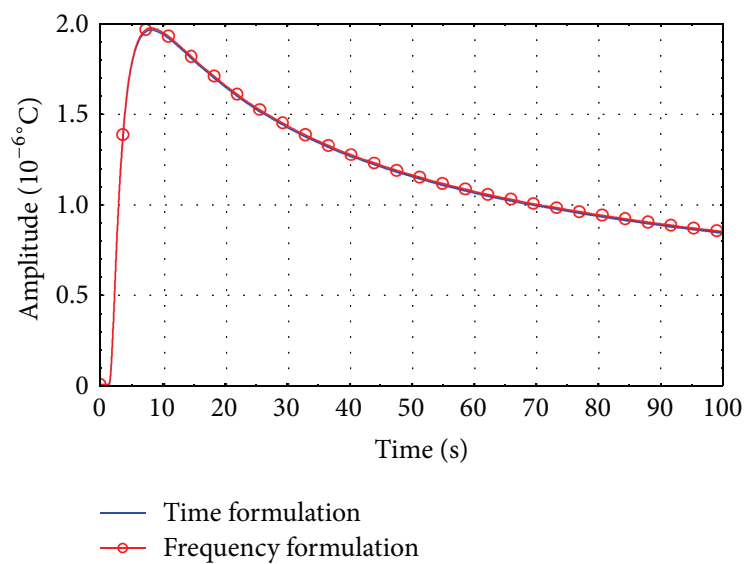

(a)

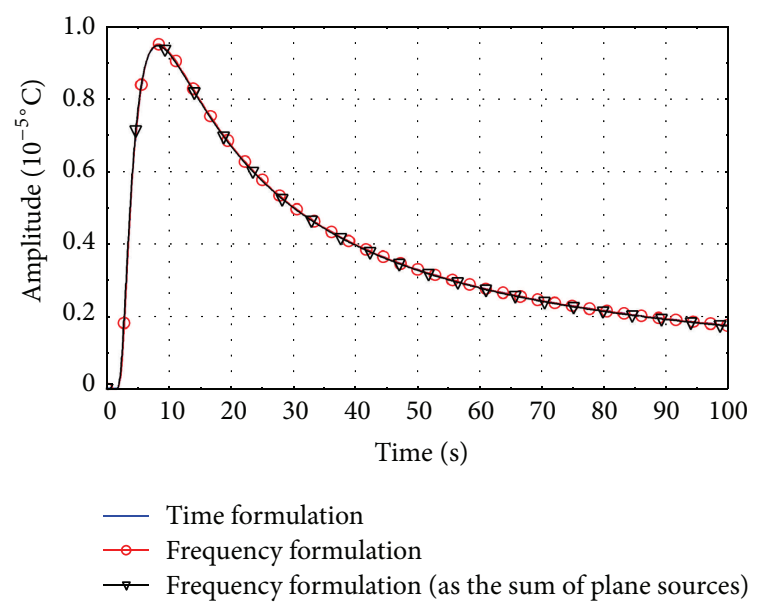

(b)

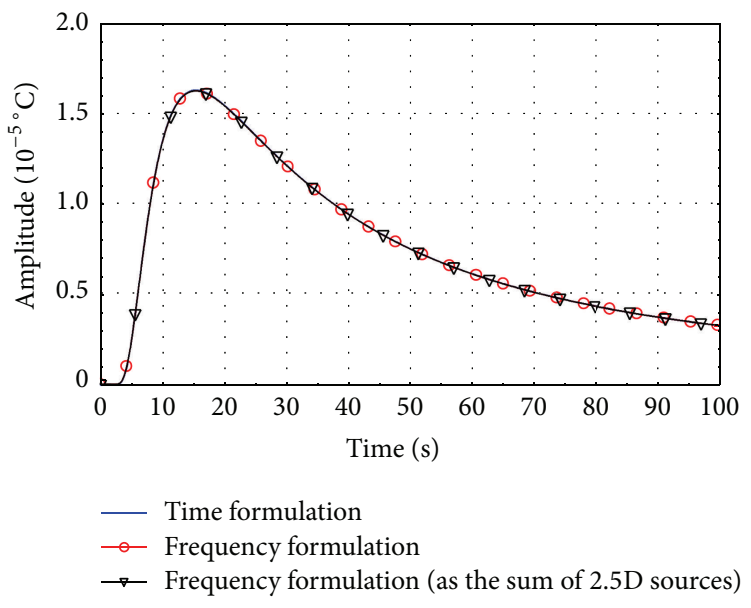

(c)

FIGURE 1: Responses for an unbounded space: (a) 1D heat source; (b) 2D heat source; (c) 3D heat source.

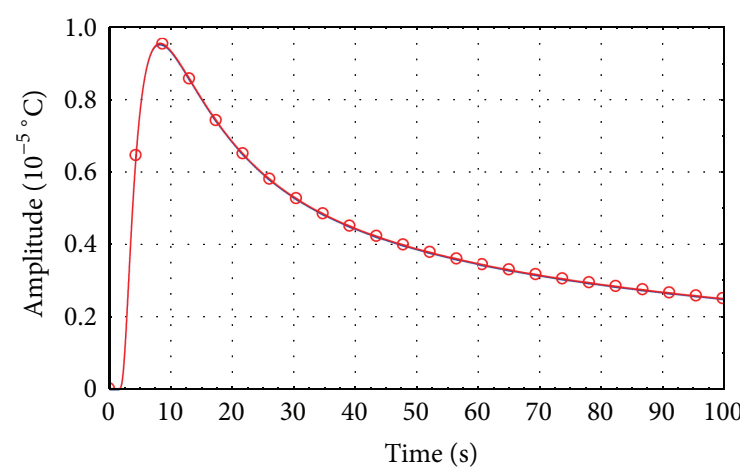

- Time formulation

$\rightarrow$ Frequency formulation

(a)

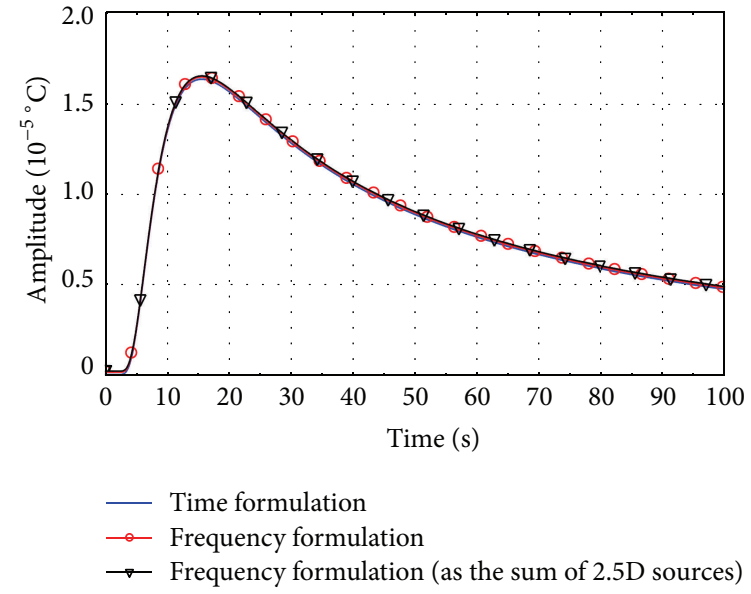

(b)

FIgURE 2: Response for the half-space (Case 1): (a) 2D heat source; (b) 3D heat source. 


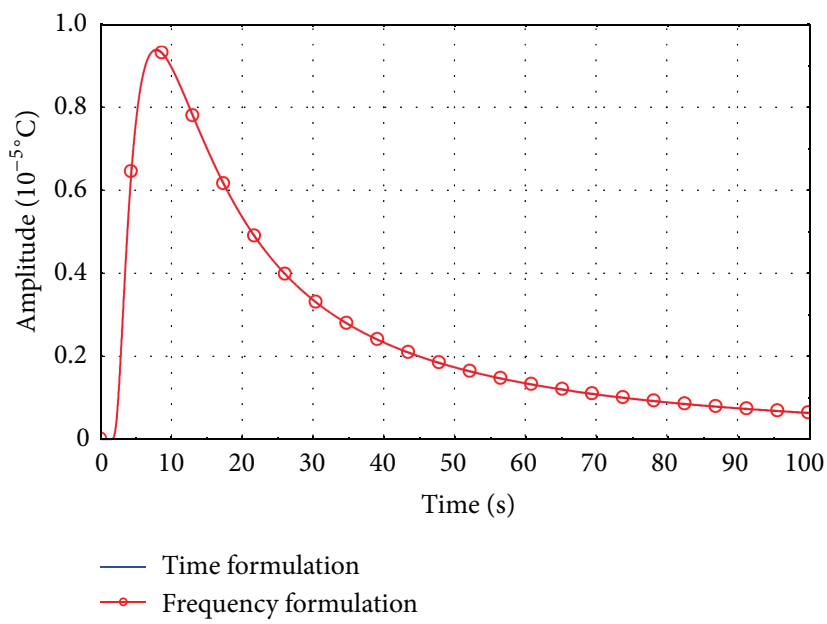

(a)

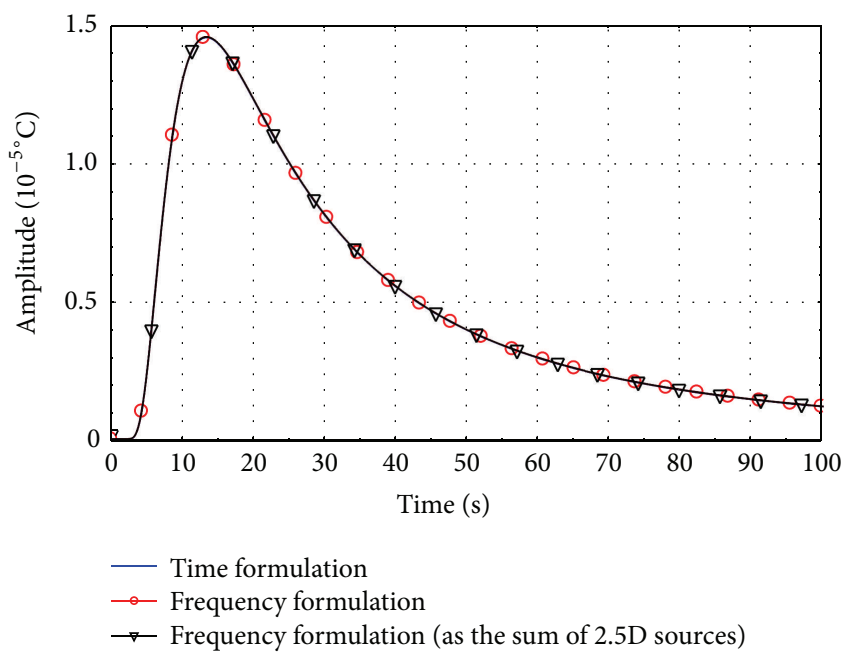

(b)

Figure 3: Response for the bounded space defined by $x \leq d_{1}$ and $y \geq 0$ (Case 3): (a) 2D heat source; (b) 3D heat source.

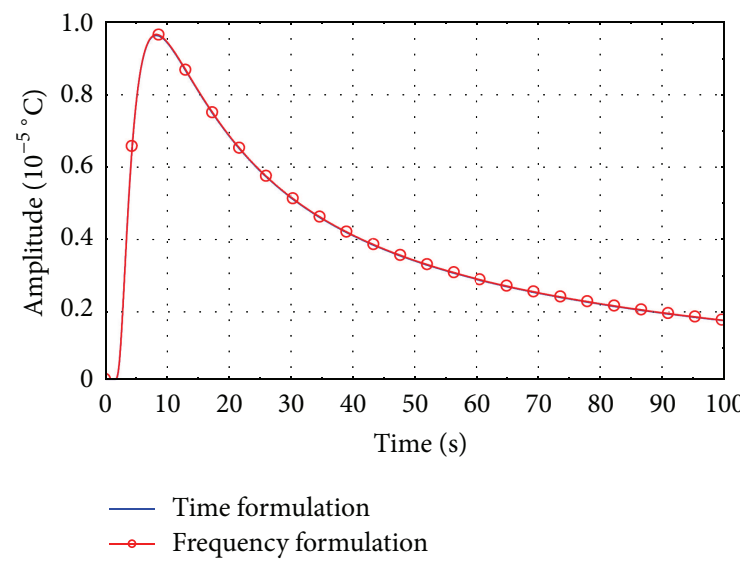

(a)

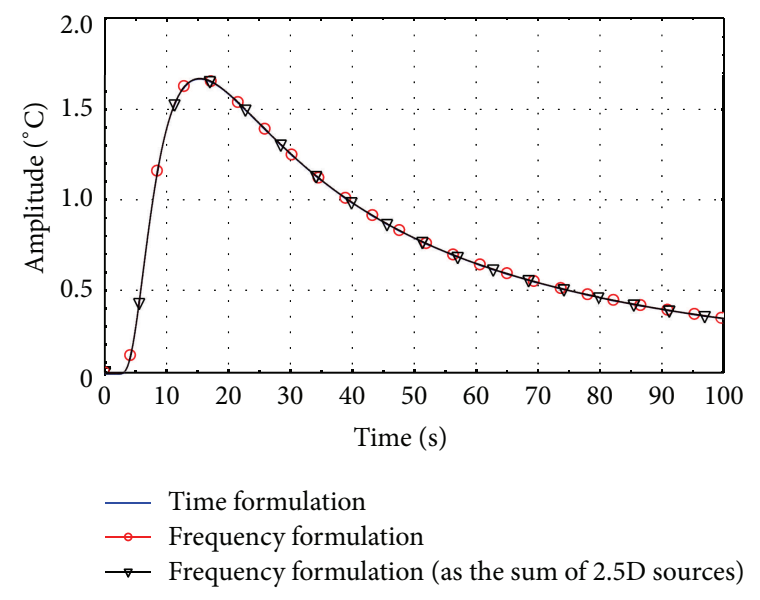

(b)

FIGURE 4: Response for the horizontal layer (Case 3): (a) 2D heat source; (b) 3D heat source.

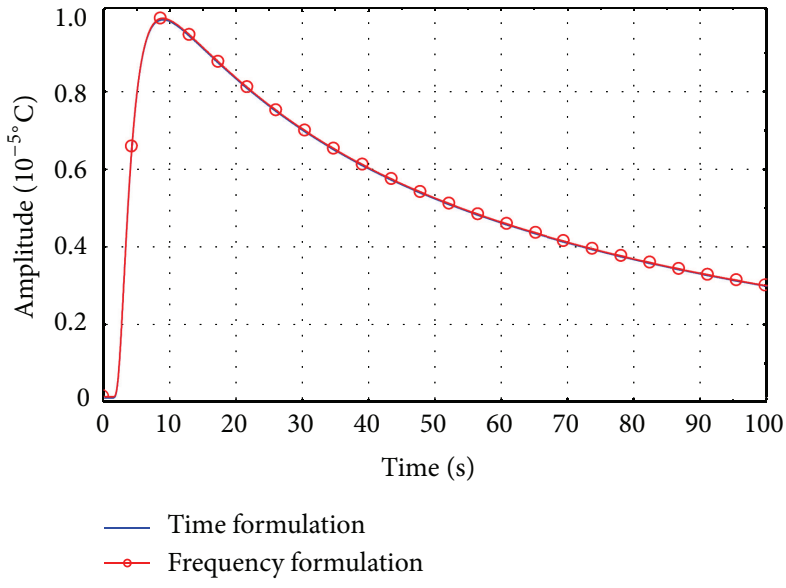

(a)

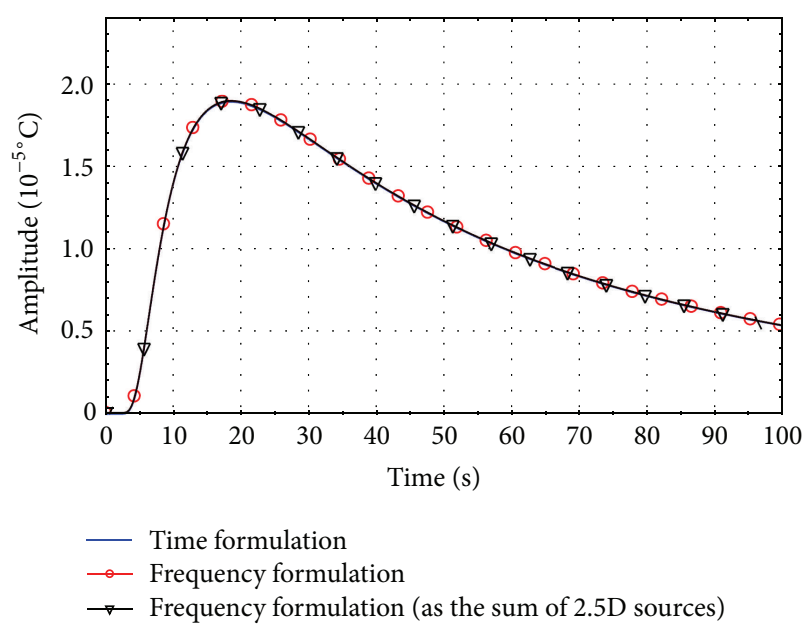

(b)

FIGURE 5: Response for the U system (Case 3): (a) 2D heat source; (b) 3D heat source. 


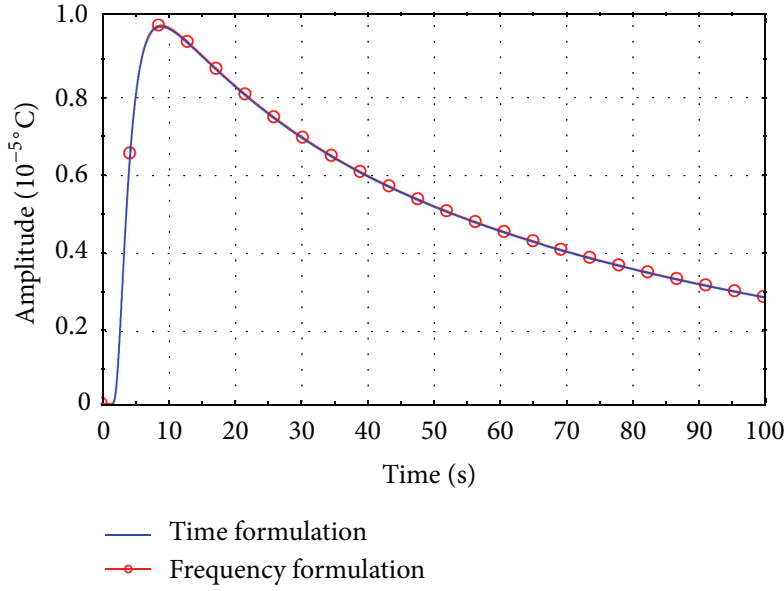

(a)

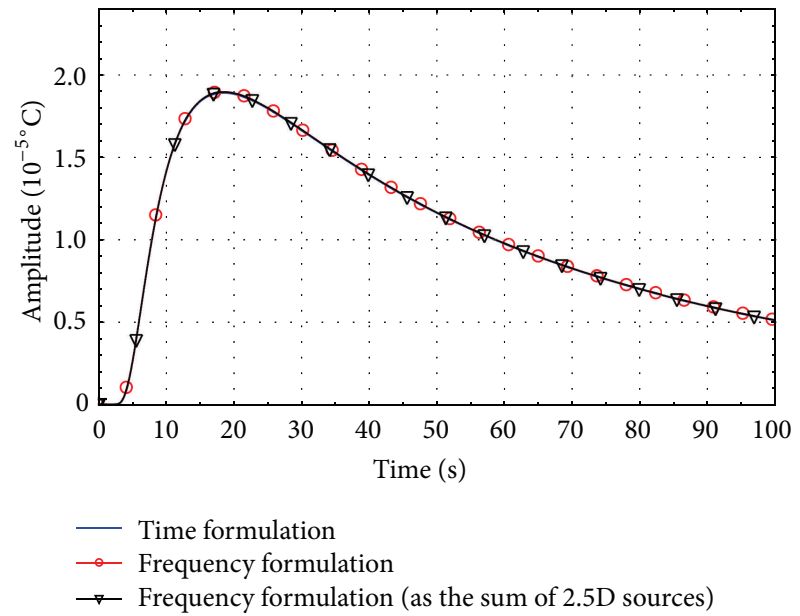

(b)

Figure 6: Response for the solid rectangular pipe (Case 3): (a) 2D heat source; (b) 3D heat source.

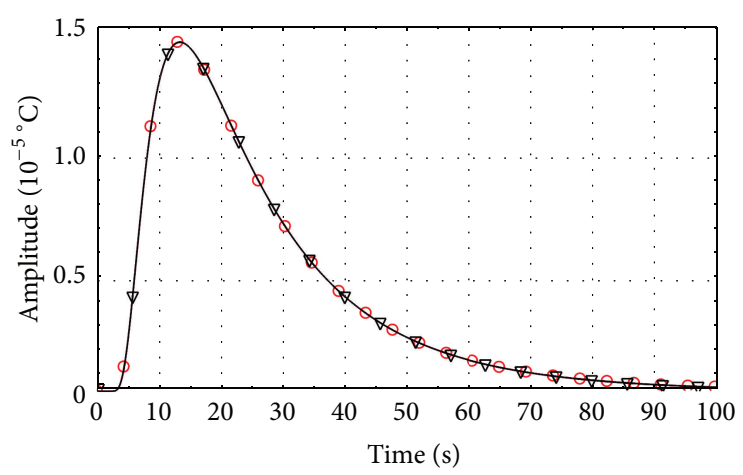

— Time formulation

$\because$ Frequency formulation

$\rightarrow$ Frequency formulation (as the sum of $2.5 \mathrm{D}$ sources)

(a)

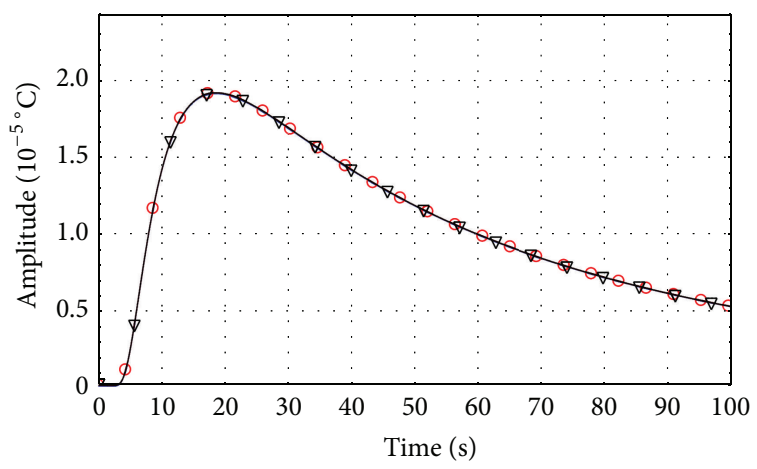

_ Time formulation

$\therefore$ Frequency formulation

$\rightarrow$ Frequency formulation (as the sum of $2.5 \mathrm{D}$ sources)

(b)

Figure 7: Response for the solid open box: (a) Case 2; (b) Case 3.

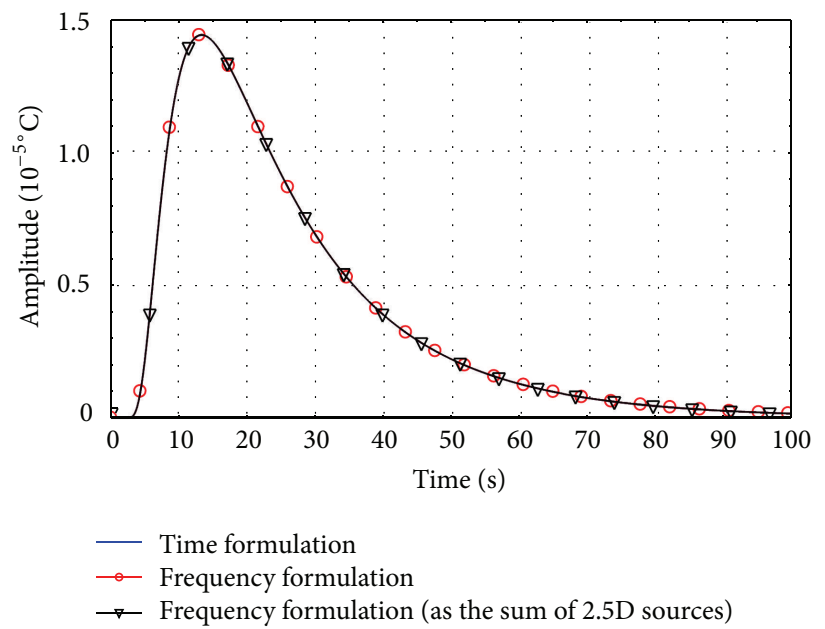

(a)

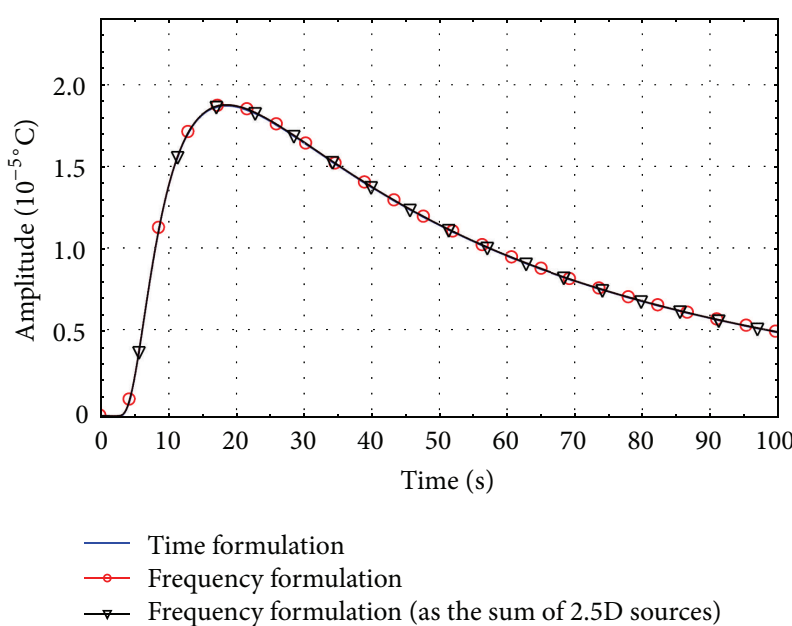

(b)

FIgURE 8: Responses for the 3D parallelepiped box: (a) Case 2. (b) Case 3. 


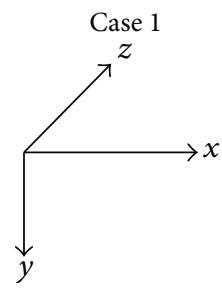

Figure 9: Unbounded space.

$2 \mathrm{D}$ source is as follows:

$$
\begin{aligned}
T(x, y, t) & =\frac{e^{-\left(r_{00}^{00}\right)^{2} / 4 K \tau}}{\rho c(4 \pi K \tau)}, \\
T(x, y, \omega) & =\frac{-i}{4 \lambda} H_{0}\left(k_{1} r_{00}^{00}\right), \\
T(x, y, \omega) & =\frac{-i}{2 \lambda L_{x}} \sum_{n_{x}=-\infty}^{\infty}\left[\frac{e^{-i k_{1 n}\left|\bar{y}_{0}\right|}}{k_{1 n}} e^{-i k_{n}\left(\bar{x}_{0}\right)}\right]
\end{aligned}
$$

as the sum of plane sources.

3D source is as follows:

$$
\begin{aligned}
T(x, y, z, t) & =\frac{e^{-\left(r_{000}^{000}\right)^{2} / 4 K \tau}}{\rho c(4 \pi K \tau)^{1.5}} \text { with } \tau=t-t_{0}, \\
T(x, y, z, \omega) & =\frac{1}{2 \lambda r_{000}^{000}} e^{-\sqrt{i \omega / K} r_{000}^{000}}, \\
T(x, y, z, \omega) & =\frac{-i \pi}{2 \lambda L_{z}} \sum_{m_{k}=-\infty}^{\infty}\left[H_{0}\left(k_{1 z} r_{00}^{00}\right) e^{-i k_{z}\left(\bar{z}_{0}\right)}\right]
\end{aligned}
$$

as the sum of $2.5 \mathrm{D}$ sources.

\subsection{Two-Dimensional Space}

(a) Half-Space Defined by $y \geq 0$. Boundary conditions prescribed for the half-space (Cases 1 and 2) are shown in Figure 10

Equations for the half-space (Cases 1 and 2), subjected to a $2 \mathrm{D}$ heat source, are as follows.

Case 1. Consider

$$
\begin{aligned}
& T(x, y, t)=\frac{1}{\rho c(4 \pi K \tau)} \sum_{j=0}^{1} e^{-\left(r_{0 j}^{00}\right)^{2} / 4 K \tau}, \\
& T(x, y, \omega)=\frac{-i}{4 \lambda} \sum_{j=0}^{1} H_{0}\left(k_{1} r_{0 j}^{00}\right) .
\end{aligned}
$$

Case 2. Consider

$$
\begin{aligned}
& T(x, y, t)=\frac{1}{\rho c(4 \pi K \tau)} \sum_{j=0}^{1}\left[(-1)^{j} e^{-\left(r_{0 j}^{00}\right)^{2} / 4 K \tau}\right], \\
& T(x, y, \omega)=\frac{-i}{4 \lambda} \sum_{j=0}^{1}\left[(-1)^{j} H_{0}\left(k_{1} r_{0 j}^{00}\right)\right] .
\end{aligned}
$$

Equations for the half-space (Cases 1 and 2), subjected to a $3 \mathrm{D}$ heat source, are as follows.

Case 1. Consider

$$
T(x, y, z, t)=\frac{1}{\rho c(4 \pi K \tau)^{1.5}} \sum_{j=0}^{1} e^{-\left(r_{0 j 0}^{000}\right)^{2} / 4 K \tau},
$$

$$
T(x, y, z, \omega)=\frac{1}{2 \lambda} \sum_{j=0}^{1} \frac{e^{-\sqrt{i \omega / K} r_{0 j 0}^{000}}}{r_{0 j 0}^{000}},
$$

$$
T(x, y, z, \omega)=\frac{-i \pi}{2 \lambda L_{x}} \sum_{j=0}^{1} \sum_{m_{k}=-\infty}^{\infty}\left[H_{0}\left(k_{1 z} r_{0 j}^{00}\right) e^{-i k_{z}\left(\bar{z}_{0}\right)}\right]
$$

as the sum of $2.5 \mathrm{D}$ sources.

Case 2. Consider

$$
\begin{aligned}
& T(x, y, z, t)=\frac{1}{\rho c(4 \pi K \tau)^{1.5}} \sum_{j=0}^{1}\left[(-1)^{j} e^{-\left(r_{0 j 0}^{000}\right)^{2} / 4 K \tau}\right], \\
& T(x, y, z, \omega)=\frac{1}{2 \lambda} \sum_{j=0}^{1}\left[(-1)^{j} \frac{e^{-\sqrt{i \omega / K} r_{0 j 0}^{000}}}{r_{0 j 0}^{000}}\right], \\
& T(x, y, z, \omega) \\
& \quad=\frac{-i \pi}{2 \lambda L_{x}} \sum_{j=0}^{1}(-1)^{j} \sum_{m_{k}=-\infty}^{\infty}\left[H_{0}\left(k_{1 z} r_{0 j}^{00}\right) e^{-i k_{z}\left(\bar{z}_{0}\right)}\right]
\end{aligned}
$$

as the sum of $2.5 \mathrm{D}$ sources.

(b) Bounded Space Defined by $x \leq d_{1}$ and $y \geq 0$. Boundary conditions prescribed for the bounded space defined by $x \leq$ $d_{1}$ and $y \geq 0$ (Cases 1-4) are shown in Figure 11 .

Equations for the bounded space defined by $x \leq d_{1}$ and $y \geq 0$ (Cases 1-4), subjected to a $2 \mathrm{D}$ heat source, are as follows.

Case 1. Consider

$$
\begin{aligned}
& T(x, y, t)=\frac{1}{\rho c(4 \pi K \tau)} \sum_{i=0}^{1} \sum_{j=0}^{1}\left[e^{-\left(r_{i j}^{i 0}\right)^{2} / 4 K \tau}\right], \\
& T(x, y, \omega)=\frac{-i}{4 \lambda} \sum_{i=0}^{1} \sum_{j=0}^{1}\left[H_{0}\left(k_{1} r_{i j}^{i 0}\right)\right] .
\end{aligned}
$$

\section{Case 2. Consider}

$$
\begin{aligned}
& T(x, y, t)=\frac{1}{\rho c(4 \pi K \tau)} \sum_{i=0}^{1} \sum_{j=0}^{1}\left[(-1)^{(i+j)} e^{-\left(r_{i j}^{i 0}\right)^{2} / 4 K \tau}\right], \\
& T(x, y, \omega)=\frac{-i}{4 \lambda} \sum_{i=0}^{1} \sum_{j=0}^{1}\left[(-1)^{(i+j)} H_{0}\left(k_{1} r_{i j}^{i 0}\right)\right] .
\end{aligned}
$$



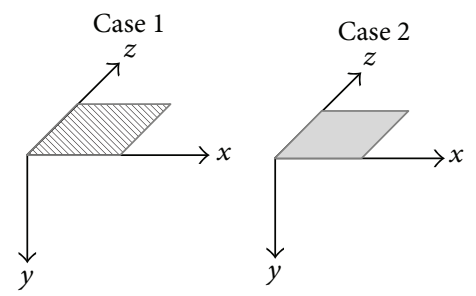

$\mathbb{Q}$ Null heat fluxes

$\square$ Null temperatures

(a)

(b)

FIGURE 10: Boundary conditions prescribed for the half-space (Cases 1 and 2).
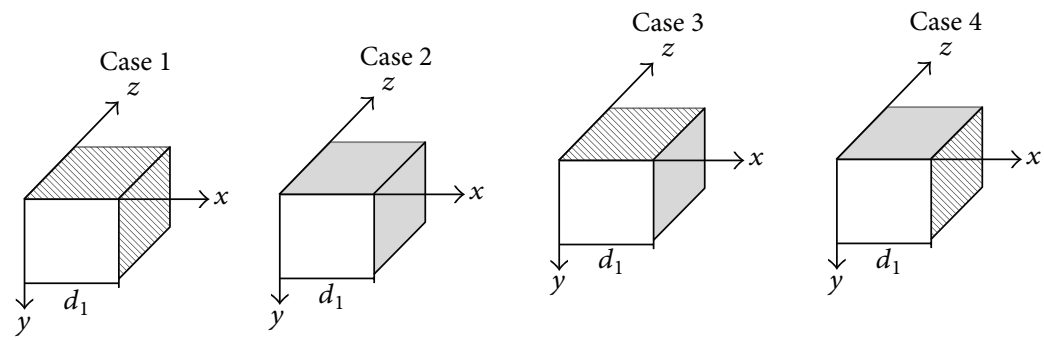

$\mathbb{Q}$ Null heat fluxes

$\square$ Null temperatures

$\mathbb{Q}$ Null heat fluxes

$\mathbb{N}$ Null heat fluxes $\square$ Null temperatures

$\square$ Null temperatures

(a)

(b)

(c)

(d)

FIGURE 11: Boundary conditions prescribed for the bounded space defined by $x \leq d_{1}$ and $y \geq 0$ (Cases 1-4).

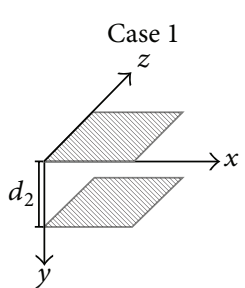

$\mathbb{N}$ Null heat fluxes

(a)

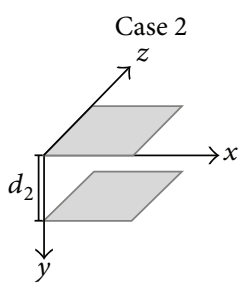

$\square$ Null temperatures

(b)

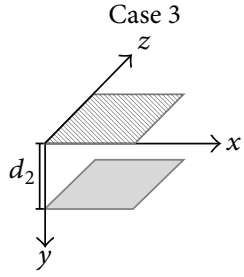

$\mathbb{Q}$ Null heat fluxes

$\square$ Null temperatures

(c)

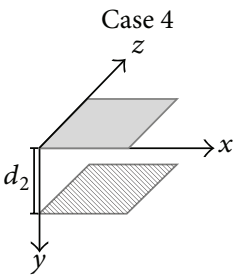

$\mathbb{Q}$ Null heat fluxes

$\square$ Null temperatures

(d)

FiguRE 12: Boundary conditions prescribed for the horizontal layer (Cases 1-4).

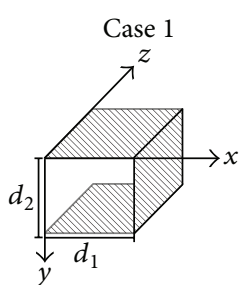

$\mathbb{N}$ Null heat fluxes

(a)

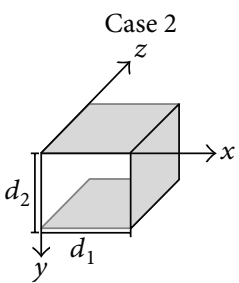

Null temperatures

(b)

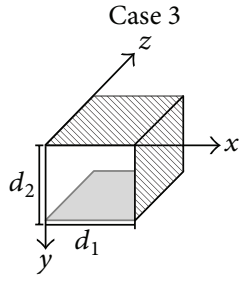

$\mathbb{Q}$ Null heat fluxes Null temperatures

(c)

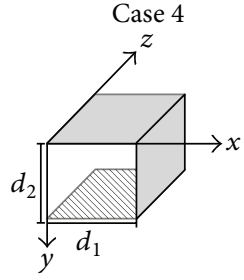

$\mathbb{Q}$ Null heat fluxes

$\square$ Null temperatures

(d)

FIGURE 13: Boundary conditions prescribed for the U system (Cases 1-4). 


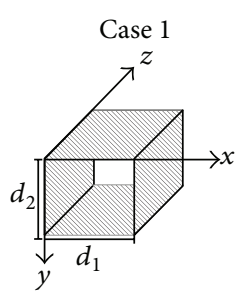

$\mathbb{N}$ Null heat fluxes

(a)

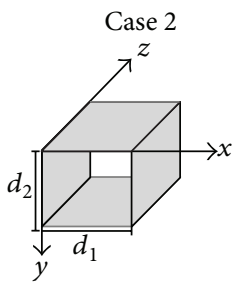

Null temperatures

(b)

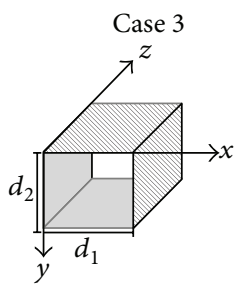

$\mathbb{8}$ Null heat fluxes

$\square$ Null temperatures

(c)

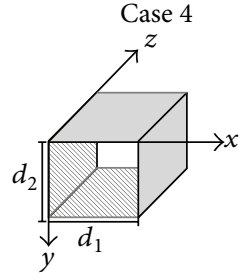

$\mathbb{Q}$ Null heat fluxes

$\square$ Null temperatures

(d)

FIGURE 14: Boundary conditions prescribed for the solid rectangular pipe (Cases 1-4).

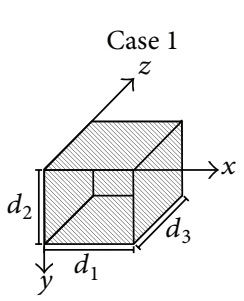

$\mathbb{N}$ Null heat fluxes

(a)

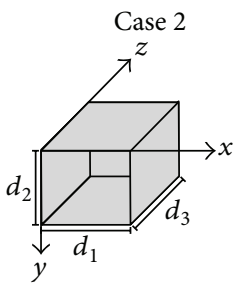

$\square$ Null temperatures

(b)

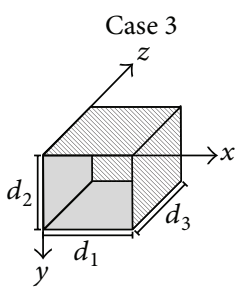

Null heat fluxes $\square$ Null temperatures

(c)

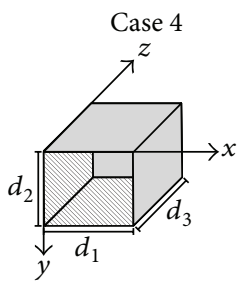

Null heat fluxes

Null temperatures

(d)

FIGURE 15: Boundary conditions prescribed for the solid open box (Cases 1-4).

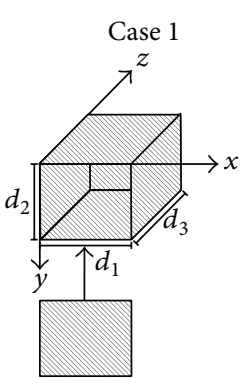

$\mathbb{N}$ Null heat fluxes

(a)

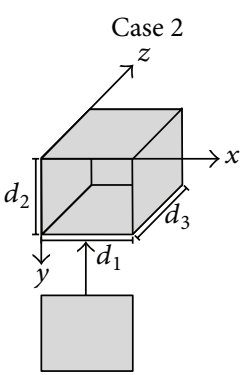

Null temperatures

(b)

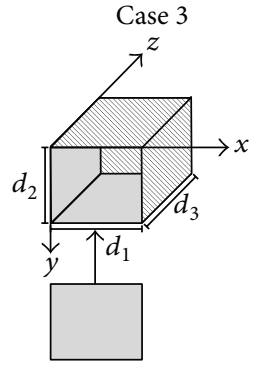

Null heat fluxes $\square$ Null temperatures

(c)

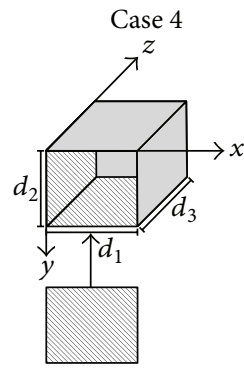

Null heat fluxes Null temperatures

(d)

FIGURE 16: Boundary conditions for the 3D parallelepiped box (Cases 1-4).

Case 3. Consider

$$
\begin{aligned}
& T(x, y, t)=\frac{1}{\rho c(4 \pi K \tau)} \sum_{i=0}^{1} \sum_{j=0}^{1}\left[(-1)^{i} e^{-\left(r_{i j}^{i 0}\right)^{2} / 4 K \tau}\right], \\
& T(x, y, \omega)=\frac{-i}{4 \lambda} \sum_{i=0}^{1} \sum_{j=0}^{1}\left[(-1)^{i} H_{0}\left(k_{1} r_{i j}^{i 0}\right)\right] .
\end{aligned}
$$

Case 4. Consider

$$
\begin{aligned}
& T(x, y, t)=\frac{1}{\rho c(4 \pi K \tau)} \sum_{i=0}^{1} \sum_{j=0}^{1}\left[(-1)^{j} e^{-\left(r_{i j}^{i 0}\right)^{2} / 4 K \tau}\right], \\
& T(x, y, \omega)=\frac{-i}{4 \lambda} \sum_{i=0}^{1} \sum_{j=0}^{1}\left[(-1)^{j} H_{0}\left(k_{1} r_{i j}^{i 0}\right)\right] .
\end{aligned}
$$


Equations defined for the bounded space defined by $x \leq$ $d_{1}$ and $y \geq 0$ (Cases 1-4) and subjected to a 3D heat source, are as follows.

Case 1. Consider

$$
\begin{aligned}
& T(x, y, z, t)=\frac{1}{\rho c(4 \pi K \tau)^{1.5}} \sum_{i=0}^{1} \sum_{j=0}^{1}\left[e^{-\left(r_{i j 0}^{i 00}\right)^{2} / 4 K \tau}\right], \\
& T(x, y, z, \omega)=\frac{1}{2 \lambda} \sum_{i=0}^{1} \sum_{j=0}^{1}\left[(-1)^{(i+j)} \frac{e^{-\sqrt{i \omega / K} r_{i j 0}^{i 00}}}{r_{i j 0}^{i 00}}\right], \\
& T(x, y, z, \omega) \\
& =\frac{-i \pi}{2 \lambda L_{x}} \sum_{i=0}^{1} \sum_{j=0}^{1} \sum_{m_{k}=-\infty}^{\infty}\left[H_{0}\left(k_{1 z} r_{i j}^{10}\right) e^{-i k_{z}\left(\bar{z}_{0}\right)}\right]
\end{aligned}
$$

as the sum of $2.5 \mathrm{D}$ sources.

Case 2. Consider

$$
\begin{aligned}
& T(x, y, z, t) \\
& =\frac{1}{\rho c(4 \pi K \tau)^{1.5}} \sum_{i=0}^{1} \sum_{j=0}^{1}\left[(-1)^{(i+j)} e^{-\left(r_{i j 0}^{i 00}\right)^{2} / 4 K \tau}\right] \\
& T(x, y, z, \omega)=\frac{1}{2 \lambda} \sum_{i=0}^{1} \sum_{j=0}^{1}\left[(-1)^{(i+j)} \frac{e^{-\sqrt{i \omega / K} r_{i j 0}^{i 00}}}{r_{i j 0}^{i 00}}\right], \\
& T(x, y, z, \omega) \\
& \quad=\frac{-i \pi}{2 \lambda L_{x}} \sum_{i=0}^{1} \sum_{j=0}^{1}(-1)^{(i+j)} \sum_{m_{k}=-\infty}^{\infty}\left[H_{0}\left(k_{1 z^{i j}} r_{i j}^{i 0}\right) e^{-i k_{z}\left(\bar{z}_{0}\right)}\right]
\end{aligned}
$$$$
\text { as the sum of } 2.5 \mathrm{D} \text { sources. }
$$

Case 3. Consider

$$
\begin{aligned}
& T(x, y, z, t) \\
& =\frac{1}{\rho c(4 \pi K \tau)^{1.5}} \sum_{i=0}^{1} \sum_{j=0}^{1}\left[(-1)^{i} e^{-\left(r_{i j 0}^{i 00}\right)^{2} / 4 K \tau}\right], \\
& T(x, y, z, \omega)=\frac{1}{2 \lambda} \sum_{i=0}^{1} \sum_{j=0}^{1}\left[(-1)^{i} \frac{e^{-\sqrt{i \omega / K} r_{i j 0}^{i 00}}}{r_{i j 0}^{i 00}}\right], \\
& T(x, y, z, \omega) \\
& \quad=\frac{-i \pi}{2 \lambda L_{x}} \sum_{i=0}^{1} \sum_{j=0}^{1}(-1)^{(i)} \sum_{m_{k}=-\infty}^{\infty}\left[H_{0}\left(k_{1 z} r_{i j}^{i 0}\right) e^{-i k_{z}\left(\bar{z}_{0}\right)}\right]
\end{aligned}
$$

as the sum of $2.5 \mathrm{D}$ sources.
Case 4. Consider

$$
T(x, y, z, t)
$$

$$
\begin{aligned}
& =\frac{1}{\rho c(4 \pi K \tau)^{1.5}} \sum_{i=0}^{1} \sum_{j=0}^{1}\left[(-1)^{j} e^{-\left(r_{i j 0}^{i 00}\right)^{2} / 4 K \tau}\right] \\
& T(x, y, z, \omega)=\frac{1}{2 \lambda} \sum_{i=0}^{1} \sum_{j=0}^{1}\left[(-1)^{j} \frac{e^{-\sqrt{i \omega / K} r_{i j 0}^{i 00}}}{r_{i j 0}^{i 00}}\right], \\
& T(x, y, z, \omega) \\
& \quad=\frac{-i \pi}{2 \lambda L_{x}} \sum_{i=0}^{1} \sum_{j=0}^{1}(-1)^{(j)} \sum_{m_{k}=-\infty}^{\infty}\left[H_{0}\left(k_{1 z} r_{i j}^{i 0}\right) e^{-i k_{z}\left(\bar{z}_{0}\right)}\right]
\end{aligned}
$$

as the sum of $2.5 \mathrm{D}$ sources.

(c) Horizontal Layer Defined by $y=0$ and $y=d_{2}$. Boundary conditions prescribed for the horizontal layer (Cases 1-4) are shown in Figure 12.

Equations for the horizontal layer (Cases 1-4), subjected to a $2 \mathrm{D}$ heat source, are as follows.

Case 1. Consider

$$
\begin{aligned}
& T(x, y, t) \\
& =\frac{1}{\rho c(4 \pi K \tau)}\left[e^{-\left(r_{00}^{00}\right)^{2} / 4 K \tau}+\sum_{m=1}^{N S_{y}} \sum_{j=1}^{4} e^{-\left(r_{0 j}^{0 m}\right)^{2} / 4 K \tau}\right], \\
& T(x, y, \omega)=\frac{-i}{4 \lambda}\left[H_{0}\left(k_{1} r_{00}^{00}\right)+\sum_{m=1}^{N S_{y}} \sum_{j=1}^{4} H_{0}\left(k_{1} r_{0 j}^{0 m}\right)\right] .
\end{aligned}
$$

Case 2. Consider

$$
\begin{gathered}
T(x, y, t)=\frac{1}{\rho c(4 \pi K \tau)}\left[e^{-\left(r_{00}^{00}\right)^{2} / 4 K \tau}\right. \\
\left.+\sum_{m=1}^{N S_{y}} \sum_{j=1}^{4}(-1)^{(j-1) !} e^{-\left(r_{0 j}^{0 m}\right)^{2} / 4 K \tau}\right], \\
T(x, y, \omega)=\frac{-i}{4 \lambda}\left[H_{0}\left(k_{1} r_{00}^{00}\right)\right. \\
\left.+\sum_{m=1}^{N S_{y}} \sum_{j=1}^{4}(-1)^{(j-1) !} H_{0}\left(k_{1} r_{0 j}^{0 m}\right)\right] .
\end{gathered}
$$


Case 3. Consider

$$
\begin{gathered}
T(x, y, t)=\frac{1}{\rho c(4 \pi K \tau)}\left[e^{-\left(r_{00}^{00}\right)^{2} / 4 K \tau}+\sum_{m=1}^{N S_{y}}(-1)^{(m-1)}\right. \\
\left.\cdot\left[\sum_{j=1}^{4}(-1)^{((j-1) !(j+1)+1)} e^{-\left(r_{0 j}^{0 m}\right)^{2} / 4 K \tau}\right]\right] \\
T(x, y, \omega)=\frac{-i}{4 \lambda}\left[H_{0}\left(k_{1} r_{00}^{00}\right)+\sum_{m=1}^{N S_{y}}(-1)^{(m-1)}\right. \\
\cdot\left[\sum_{j=1}^{4}(-1)^{((j-1) !(j+1)+1)} H_{0}\left(k_{1} r_{0 j}^{0 m}\right)\right]
\end{gathered}
$$

Case 4. Consider

$$
\begin{aligned}
& T(x, y, t)=\frac{1}{\rho c(4 \pi K \tau)}\left[e^{-\left(r_{00}^{00}\right)^{2} / 4 K \tau}\right. \\
& \left.+\sum_{m=1}^{N S_{y}}(-1)^{(m-1)}\left[\sum_{j=1}^{4}(-1)^{(j !+1)} e^{-\left(r_{0 j}^{0 m}\right)^{2} / 4 K \tau}\right]\right], \\
& T(x, y, \omega)=\frac{-i}{4 \lambda}\left[H_{0}\left(k_{1} r_{00}^{00}\right)\right. \\
& \left.+\sum_{m=1}^{N S_{y}}(-1)^{(m-1)}\left[\sum_{j=1}^{4}(-1)^{(j !+1)} H_{0}\left(k_{1} r_{0 j}^{0 m}\right)\right]\right] .
\end{aligned}
$$

Equations for the horizontal layer (Cases 1-4), subjected to a $3 \mathrm{D}$ heat source, are as follows.

\section{Case 1. Consider}

$$
\begin{aligned}
& T(x, y, z, t)=\frac{1}{\rho c(4 \pi K \tau)^{1.5}}\left[e^{-\left(r_{000}^{000}\right)^{2} / 4 K \tau}\right. \\
& \left.+\sum_{m=1}^{N S_{y}} \sum_{j=1}^{4} e^{-\left(r_{0 j 0}^{0 m 0}\right)^{2} / 4 K \tau}\right] \\
& T(x, y, z, \omega)=\frac{1}{2 \lambda}\left[\frac{e^{-\sqrt{i \omega / K} r_{000}^{000}}}{r_{000}^{000}}\right. \\
& \left.+\sum_{m=1}^{N S_{y}} \sum_{j=1}^{4} \frac{e^{-\sqrt{i \omega / K} r_{0 j 0}^{0 m 0}}}{r_{0 j 0}^{0 m 0}}\right]
\end{aligned}
$$

$$
\begin{aligned}
& T(x, y, z, \omega)=\frac{-i \pi}{2 \lambda L_{x}}\left[\sum_{m_{k}=-\infty}^{\infty}\left[H_{0}\left(k_{1 z} r_{00}^{00}\right) e^{-i k_{z}\left(\bar{z}_{0}\right)}\right]\right. \\
& \left.\quad+\sum_{m=1}^{N S_{y}} \sum_{j=1}^{4} \sum_{m_{k}=-\infty}^{\infty}\left[H_{0}\left(k_{1 z} r_{0 j}^{0 m}\right) e^{-i k_{z}\left(\bar{z}_{0}\right)}\right]\right]
\end{aligned}
$$$$
\text { as the sum of } 2.5 \mathrm{D} \text { sources. }
$$

Case 2. Consider

$$
\begin{aligned}
& T(x, y, z, t)=\frac{1}{\rho c(4 \pi K \tau)^{1.5}}\left[e^{-\left(r_{000}^{000}\right)^{2} / 4 K \tau}\right. \\
& \left.+\sum_{m=1}^{N S_{y}} \sum_{j=1}^{4}(-1)^{(j-1) !} e^{-\left(r_{0 j 0}^{0 m 0}\right)^{2} / 4 K \tau}\right], \\
& T(x, y, z, \omega)=\frac{1}{2 \lambda}\left[\frac{e^{-\sqrt{i \omega / K} r_{000}^{000}}}{r_{000}^{000}}\right. \\
& \left.+\sum_{m=1}^{N S_{y}} \sum_{j=1}^{4}(-1)^{(j-1) !} \frac{e^{-\sqrt{i \omega / K} r_{0 j 0}^{0 m 0}}}{r_{0 j 0}^{0 m 0}}\right], \\
& T(x, y, z, \omega)=\frac{-i \pi}{2 \lambda L_{x}}\left[\sum_{m_{k}=-\infty}^{\infty}\left[H_{0}\left(k_{1 z} r_{00}^{00}\right) e^{-i k_{z}\left(\bar{z}_{0}\right)}\right]\right. \\
& \quad+\sum_{m=1}^{N S_{y}} \sum_{j=1}^{4}(-1)^{(j-1) !} \sum_{m_{k}=-\infty}^{\infty}\left[H_{0}\left(k_{1 z} r_{0 j}^{0 m}\right) e^{-i k_{z}\left(\bar{z}_{0}\right)}\right]
\end{aligned}
$$

Case 3. Consider

$$
\begin{aligned}
& T(x, y, z, t)=\frac{1}{\rho c(4 \pi K \tau)^{1.5}}\left[e^{-\left(r_{000}^{000}\right)^{2} / 4 K \tau}\right. \\
& \left.\quad+\sum_{m=1}^{N S_{y}}(-1)^{(m-1)} \sum_{j=1}^{4}(-1)^{((j-1) !(j+1)+1)} e^{-\left(r_{0 j 0}^{0 m 0}\right)^{2} / 4 K \tau}\right], \\
& T(x, y, z, \omega)=\frac{1}{2 \lambda}\left[\frac{e^{-\sqrt{i \omega / K} r_{000}^{000}}}{r_{000}^{000}}+\sum_{m=1}^{N S_{y}}(-1)^{(m-1)}\right. \\
& \left.\quad \cdot \sum_{j=1}^{4}(-1)^{((j-1) !(j+1)+1)} \frac{e^{-\sqrt{i \omega / K} r_{0 j 0}^{0 m 0}}}{r_{0 j 0}^{0 m 0}}\right]
\end{aligned}
$$




$$
\begin{aligned}
& T(x, y, z, \omega)=\frac{-i \pi}{2 \lambda L_{x}}\left[\sum_{m_{k}=-\infty}^{\infty}\left[H_{0}\left(k_{1 z} r_{00}^{00}\right) e^{-i k_{z}\left(\bar{z}_{0}\right)}\right]\right. \\
& +\sum_{m=1}^{N S_{y}}(-1)^{(m-1)} \sum_{j=1}^{4}(-1)^{(j-1) !} \\
& \left.\quad \cdot \sum_{m_{k}=-\infty}^{\infty}\left[H_{0}\left(k_{1 z} r_{0 j}^{0 m}\right) e^{-i k_{z}\left(\bar{z}_{0}\right)}\right]\right]
\end{aligned}
$$

as the sum of $2.5 \mathrm{D}$ sources.

Case 4. Consider

$$
\begin{aligned}
& T(x, y, z, t)=\frac{1}{\rho c(4 \pi K \tau)^{1.5}}\left[e^{-\left(r_{000}^{000}\right)^{2} / 4 K \tau}\right] \\
& \left.+\sum_{m=1}^{N S_{y}}(-1)^{(m-1)} \sum_{j=1}^{4}(-1)^{(j !+1)} e^{-\left(r_{0 j 0}^{0 m 0}\right)^{2} / 4 K \tau}\right], \\
& T(x, y, z, \omega)=\frac{1}{2 \lambda}\left[\frac{e^{-\sqrt{i \omega / K} r_{000}^{000}}}{r_{000}^{000}}+\sum_{m=1}^{N S_{y}}(-1)^{(m-1)}\right. \\
& \left.\cdot \sum_{j=1}^{4}(-1)^{(j !+1)} \frac{e^{-\sqrt{i \omega / K} r_{0 j 0}^{0 m 0}}}{r_{0 j 0}^{0 m 0}}\right], \\
& T(x, y, z, \omega)=\frac{-i \pi}{2 \lambda L_{x}}\left[\sum_{m_{k}=-\infty}^{\infty}\left[H_{0}\left(k_{1 z} z_{00}^{00}\right) e^{-i k_{z}\left(\bar{z}_{0}\right)}\right]\right. \\
& +\sum_{m=1}^{N S_{y}}(-1)^{(m-1)} \sum_{j=1}^{4}(-1)^{(j !+1)} \\
& \left.\quad \sum_{m_{k}=-\infty}^{\infty}\left[H_{0}\left(k_{1 z} r_{0 j}^{0 m}\right) e^{-i k_{z}\left(\bar{z}_{0}\right)}\right]\right]
\end{aligned}
$$

as the sum of $2.5 \mathrm{D}$ sources.

(d) U System Bounded by $y \geq 0, y \leq d_{2}$, and $x \leq d_{1}$. Boundary conditions prescribed for the $U$ system (Cases 1-4) are shown in Figure 13.

Equations for the U system (Cases 1-4), subjected to a 2D heat source, are as follows.
Case 1. Consider

$$
T(x, y, t)
$$$$
=\frac{1}{\rho c(4 \pi K \tau)} \sum_{i=0}^{1}\left[e^{-\left(r_{i 0}^{i 0}\right)^{2} / 4 K \tau}+\sum_{m=1}^{N S_{y}} \sum_{j=1}^{4} e^{-\left(r_{i j}^{i m}\right)^{2} / 4 K \tau}\right],
$$

$T(x, y, \omega)$

$$
=\frac{-i}{4 \lambda} \sum_{i=0}^{1}\left[H_{0}\left(k_{1} r_{i 0}^{i 0}\right)+\sum_{m=1}^{N S_{y}} \sum_{j=1}^{4} H_{0}\left(k_{1} r_{i j}^{i m}\right)\right] .
$$

Case 2. Consider

$$
\begin{aligned}
& T(x, y, t)=\frac{1}{\rho c(4 \pi K \tau)} \sum_{i=0}^{1}(-1)^{i} \\
& \cdot\left[e^{-\left(r_{i 0}^{i 0}\right)^{2} / 4 K \tau}+\sum_{m=1}^{N S_{y}} \sum_{j=1}^{4}(-1)^{(j-1) !} e^{-\left(r_{i j}^{i m}\right)^{2} / 4 K \tau}\right], \\
& T(x, y, \omega)=\frac{-i}{4 \lambda} \sum_{i=0}^{1}(-1)^{i} \\
& \quad\left[H_{0}\left(k_{1} r_{i 0}^{i 0}\right)+\sum_{m=1}^{N S_{y}} \sum_{j=1}^{4}(-1)^{(j-1) !} H_{0}\left(k_{1} r_{i j}^{i m}\right)\right] .
\end{aligned}
$$

Case 3. Consider

$$
\begin{aligned}
& T(x, y, t)=\frac{1}{\rho c(4 \pi K \tau)} \sum_{i=0}^{1}\left[e^{-\left(r_{i 0}^{i 0}\right)^{2} / 4 K \tau}\right. \\
& \left.\quad+\sum_{m=1}^{N S_{y}}(-1)^{(m-1)} \sum_{j=1}^{4}(-1)^{((j-1) !(j+1)+1)} e^{-\left(r_{i j}^{i m}\right)^{2} / 4 K \tau}\right],
\end{aligned}
$$

$$
T(x, y, \omega)=\frac{-i}{4 \lambda} \sum_{i=0}^{1}\left[H_{0}\left(k_{1} r_{i 0}^{i 0}\right)\right.
$$

$$
\left.+\sum_{m=1}^{N S_{y}}(-1)^{(m-1)} \sum_{j=1}^{4}(-1)^{((j-1) !(j+1)+1)} H_{0}\left(k_{1} r_{i j}^{i m}\right)\right] \text {. }
$$


Case 4. Consider

$$
\begin{aligned}
& T(x, y, t)=\frac{1}{\rho c(4 \pi K \tau)} \sum_{i=0}^{1}(-1)^{i}\left[e^{-\left(r_{i 0}^{i 0}\right)^{2} / 4 K \tau}\right. \\
& \left.+\sum_{m=1}^{N S_{y}}(-1)^{(m-1)} \sum_{j=1}^{4}(-1)^{(j !+1)} e^{-\left(r_{i j}^{i m}\right)^{2} / 4 K \tau}\right], \\
& T(x, y, \omega)=\frac{-i}{4 \lambda} \sum_{i=0}^{1}(-1)^{i}\left[H_{0}\left(k_{1} r_{i 0}^{i 0}\right)\right. \\
& \left.+\sum_{m=1}^{N S_{y}}(-1)^{(m-1)} \sum_{j=1}^{4}(-1)^{(j !+1)} H_{0}\left(k_{1} r_{i j}^{i m}\right)\right] .
\end{aligned}
$$

Equations for the $\mathrm{U}$ system (Cases 1-4), subjected to a 3D heat source, are as follows.

Case 1. Consider

$$
\begin{aligned}
& T(x, y, z, \omega)=\frac{1}{2 \lambda} \sum_{i=0}^{1}(-1)^{i}\left[\frac{e^{-\sqrt{i \omega / K} r_{i 00}^{i 00}}}{r_{i 00}^{i 00}}\right. \\
& \left.+\sum_{m=1}^{N S_{y}} \sum_{j=1}^{4}(-1)^{(j-1) !} \frac{e^{-\sqrt{i \omega / K} r_{i j 0}^{i m 0}}}{r_{i j 0}^{i m 0}}\right], \\
& T(x, y, z, \omega)=\frac{-i \pi}{2 \lambda L_{x}} \sum_{i=0}^{1}(-1)^{i} \\
& +\left[\sum_{m_{k}=-\infty}^{\infty}\left[H_{0}\left(k_{1 z} r_{i 0}^{i 0}\right) e^{-i k_{z}\left(\bar{z}_{0}\right)}\right]\right. \\
& +\sum_{m=1}^{N S_{y}} \sum_{j=1}^{4}(-1)^{(j-1) !} \sum_{m_{k}=-\infty}^{\infty}\left[H_{0}\left(k_{1 z} r_{i j}^{i m}\right) e^{-i k_{z}\left(\bar{z}_{0}\right)}\right]
\end{aligned}
$$

as the sum of $2.5 \mathrm{D}$ sources.

$$
\begin{aligned}
& T(x, y, z, t)=\frac{1}{\rho c(4 \pi K \tau)^{1.5}} \sum_{i=0}^{1}\left[e^{-\left(r_{i 00}^{i 00}\right)^{2} / 4 K \tau}\right. \\
& \left.+\sum_{m=1}^{N S_{y}} \sum_{j=1}^{4} e^{-\left(r_{i j 0}^{i m 0}\right)^{2} / 4 K \tau}\right], \\
& T(x, y, z, \omega)=\frac{1}{2 \lambda} \sum_{i=0}^{1}\left[\frac{e^{-\sqrt{i \omega / K} r_{i 00}^{i 00}}}{r_{i 00}^{i 00}}\right. \\
& \left.+\sum_{m=1}^{N S_{y}} \sum_{j=1}^{4} \frac{e^{-\sqrt{i \omega / K} r_{i j 0}^{i m 0}}}{r_{i j 0}^{i m 0}}\right], \\
& T(x, y, z, \omega)=\frac{-i \pi}{2 \lambda L_{x}} \\
& \quad+\sum_{i=0}^{1}\left[\sum _ { m } ^ { \infty } \left[H_{k}=-\infty\right.\right. \\
& \quad+\sum_{m=1}^{N S_{y}} \sum_{j=1}^{4} \sum_{m_{k}=-\infty}^{\infty}\left[H_{0}\left(k_{10}^{i 0}\right) e^{-i k_{z}\left(\bar{z}_{0}\right)}\right] \\
& \left.\left.\quad r_{i j}^{i m}\right) e^{-i k_{z}\left(\bar{z}_{0}\right)}\right]
\end{aligned}
$$

as the sum of $2.5 \mathrm{D}$ sources.

Case 2. Consider

$$
\begin{aligned}
& T(x, y, z, t)=\frac{1}{\rho c(4 \pi K \tau)^{1.5}} \sum_{i=0}^{1}(-1)^{i}\left[e^{-\left(r_{i 00}^{i 00}\right)^{2} / 4 K \tau}\right. \\
& \left.\quad+\sum_{m=1}^{N S_{y}} \sum_{j=1}^{4}(-1)^{(j-1) !} e^{-\left(r_{i j 0}^{i m 0}\right)^{2} / 4 K \tau}\right]
\end{aligned}
$$

Case 3. Consider

$$
\begin{aligned}
& T(x, y, z, t)=\frac{1}{\rho c(4 \pi K \tau)^{1.5}} \sum_{i=0}^{1}(-1)^{i}\left[e^{-\left(r_{i 00}^{i 00}\right)^{2} / 4 K \tau}\right. \\
& \left.+\sum_{m=1}^{N S_{y}}(-1)^{(m-1)} \sum_{j=1}^{4}(-1)^{((j-1) !(j+1)+1)} e^{-\left(r_{i j 0}^{i m 0}\right)^{2} / 4 K \tau}\right] \\
& T(x, y, z, \omega)=\frac{1}{2 \lambda} \sum_{i=0}^{1}(-1)^{i}\left[\frac{e^{-\sqrt{i \omega / K} r_{i 00}^{i 00}}}{r_{i 00}^{i 00}}\right. \\
& \left.+\sum_{m=1}^{N S_{y}}(-1)^{(m-1)} \sum_{j=1}^{4}(-1)^{((j-1) !(j+1)+1)} \frac{e^{-\sqrt{i \omega / K} r_{i j 0}^{i m 0}}}{r_{i j 0}^{i m 0}}\right] \\
& T(x, y, z, \omega)=\frac{-i \pi}{2 \lambda L_{x}} \sum_{i=0}^{1}(-1)^{i} \\
& \quad\left[\sum_{m_{k}=-\infty}^{\infty}\left[H_{0}\left(k_{1 z^{2}} r_{i 0}^{i 0}\right) e^{-i k_{z}\left(\bar{z}_{0}\right)}\right]+\sum_{m=1}^{N S_{y}}(-1)^{(m-1)}\right.
\end{aligned}
$$$$
\cdot \sum_{j=1}^{4}(-1)^{((j-1) !(j+1)+1)}
$$$$
\left.\cdot \sum_{m_{k}=-\infty}^{\infty}\left[H_{0}\left(k_{1 z} r_{i j}^{i m}\right) e^{-i k_{z}\left(\bar{z}_{0}\right)}\right]\right]
$$

as the sum of $2.5 \mathrm{D}$ sources. 
Case 4. Consider

$$
\begin{aligned}
& T(x, y, z, t)=\frac{1}{\rho c(4 \pi K \tau)^{1.5}} \sum_{i=0}^{1}(-1)^{i}\left[e^{-\left(r_{i 00}^{i 00}\right)^{2} / 4 K \tau}\right. \\
& \left.+\sum_{m=1}^{N S_{y}}(-1)^{(m-1)} \sum_{j=1}^{4}(-1)^{(j !+1)} e^{-\left(r_{i j 0}^{i m 0}\right)^{2} / 4 K \tau}\right], \\
& T(x, y, z, \omega)=\frac{1}{2 \lambda} \sum_{i=0}^{1}(-1)^{i}\left[\frac{e^{-\sqrt{i \omega / K} r_{i 00}^{i 00}}}{r_{i 00}^{i 00}}\right. \\
& \left.+\sum_{m=1}^{N S_{y}}(-1)^{(m-1)} \sum_{j=1}^{4}(-1)^{(j !+1)} \frac{e^{-\sqrt{i \omega / K} r_{i j 0}^{i m 0}}}{r_{i j 0}^{i m 0}}\right], \\
& T(x, y, z, \omega)=\frac{-i \pi}{2 \lambda L_{x}} \sum_{i=0}^{1}(-1)^{i} \\
& \cdot\left[\sum_{m_{k}=-\infty}^{\infty}\left[H_{0}\left(k_{1 z} r_{i 0}^{i 0}\right) e^{-i k_{z}\left(\bar{z}_{0}\right)}\right]+\sum_{m=1}^{N S_{y}}(-1)^{(m-1)}\right. \\
& \left.\cdot \sum_{j=1}^{4}(-1)^{(j !+1)} \sum_{m_{k}=-\infty}^{\infty}\left[H_{0}\left(k_{1 z} r_{i j}^{i m}\right) e^{-i k_{z}\left(\bar{z}_{0}\right)}\right]\right]
\end{aligned}
$$

as the sum of $2.5 \mathrm{D}$ sources.

(e) Solid Rectangular Pipe Defined by $y \geq 0, y \leq d_{2}, x \geq 0$, and $x \leq d_{1}$. Boundary conditions prescribed for the solid rectangular pipe (Cases 1-4) are shown in Figure 14.

Equations for the solid rectangular pipe (Cases 1-4), subjected to a $2 \mathrm{D}$ heat source, are as follows.

Case 1. Consider

$$
\begin{aligned}
& T(x, y, t)=\frac{1}{\rho c(4 \pi K \tau)}\left[e^{-\left(r_{00}^{00}\right)^{2} / 4 K \tau}\right. \\
& +\sum_{m=1}^{N S_{y}} \sum_{j=1}^{4} e^{-\left(r_{0 j}^{0 m}\right)^{2} / 4 K \tau} \\
& \left.+\sum_{l=1}^{N S_{x}} \sum_{i=1}^{4}\left[e^{-\left(r_{i 0}^{l 0}\right)^{2} / 4 K \tau}+\sum_{m=1}^{N S_{y}} \sum_{j=1}^{4} e^{-\left(r_{i j}^{l m}\right)^{2} / 4 K \tau}\right]\right], \\
& T(x, y, \omega)=\frac{-i}{4 \lambda}\left[H_{0}\left(k_{1} r_{00}^{00}\right)+\sum_{m=1}^{N S_{y}} \sum_{j=1}^{4} H_{0}\left(k_{1} r_{0 j}^{0 m}\right)\right. \\
& \left.\quad+\sum_{l=1}^{N S_{x}} \sum_{i=1}^{4}\left[H_{0}\left(k_{1} r_{i 0}^{l 0}\right)+\sum_{m=1}^{N S_{y}} \sum_{j=1}^{4} H_{0}\left(k_{1} r_{i j}^{l m}\right)\right]\right] .
\end{aligned}
$$

Case 2. Consider

$$
\begin{aligned}
& T(x, y, t)=\frac{1}{\rho c(4 \pi K \tau)}\left[e^{-\left(r_{00}^{00}\right)^{2} / 4 K \tau}\right. \\
& +\sum_{m=1}^{N S_{y}} \sum_{j=1}^{4}(-1)^{(j-1) !} e^{-\left(r_{0 j}^{0 m}\right)^{2} / 4 K \tau}+\sum_{l=1}^{N S_{x}} \sum_{i=1}^{4}(-1)^{(i-1) !} \\
& \left.\cdot\left[e^{-\left(r_{i 0}^{l 0}\right)^{2} / 4 K \tau}+\sum_{m=1}^{N S_{y}} \sum_{j=1}^{4}(-1)^{(j-1) !} e^{-\left(r_{i j}^{l m}\right)^{2} / 4 K \tau}\right]\right], \\
& T(x, y, \omega)=\frac{-i}{4 \lambda}\left[H_{0}\left(k_{1} r_{00}^{00}\right)\right. \\
& +\sum_{m=1}^{N S_{y}} \sum_{j=1}^{4}(-1)^{(j-1) !} H_{0}\left(k_{1} r_{0 j}^{0 m}\right)+\sum_{l=1}^{N S_{x}} \sum_{i=1}^{4}(-1)^{(i-1) !} \\
& \left.\cdot\left[H_{0}\left(k_{1} r_{i 0}^{l 0}\right)+\sum_{m=1}^{N S_{y}} \sum_{j=1}^{4}(-1)^{(j-1) !} H_{0}\left(k_{1} r_{i j}^{i m}\right)\right]\right] \text {. }
\end{aligned}
$$

\section{Case 3. Consider}

$$
\begin{aligned}
& T(x, y, t)=\frac{1}{\rho c(4 \pi K \tau)}\left[e^{-\left(r_{00}^{00}\right)^{2} / 4 K \tau}+\sum_{m=1}^{N S_{y}}(-1)^{(m-1)}\right. \\
& \cdot \sum_{j=1}^{4}(-1)^{((j-1) !(j+1)+1)} e^{-\left(r_{0 j}^{0 m}\right)^{2} / 4 K \tau}+\sum_{l=1}^{N S_{x}}(-1)^{(l-1)} \\
& \cdot \sum_{i=1}^{4}(-1)^{(i !+1)}\left[e^{-\left(r_{i 0}^{l 0}\right)^{2} / 4 K \tau}+\sum_{m=1}^{N S_{y}}(-1)^{(m-1)}\right. \\
& \left.\left.\cdot \sum_{j=1}^{4}(-1)^{((j-1) !(j+1)+1)} e^{-\left(r_{i j}^{l m}\right)^{2} / 4 K \tau}\right]\right] \\
& T(x, y, \omega)=\frac{-i}{4 \lambda}\left[H_{0}\left(k_{1} r_{00}^{00}\right)+\sum_{m=1}^{N S_{y}}(-1)^{(m-1)}\right. \\
& \left.\cdot \sum_{j=1}^{4}(-1)^{((j-1) !(j+1)+1)} H_{0}\left(k_{1} r_{i j}^{l m}\right)\right] \\
& \cdot \sum_{i=1}^{4}(-1)^{(i !+1)}\left[H_{0}\left(k_{1} r_{i 0}^{l 0}\right)+\sum_{m=1}^{N S_{y}}(-1)^{(m-1)}\right. \\
& \quad \sum_{j=1}^{((j-1) !(j+1)+1)} H_{0}\left(k_{1} r_{0 j}^{0 m}\right)+\sum_{l=1}^{N S_{x}}(-1)^{(l-1)}
\end{aligned}
$$


Case 4. Consider

$$
\begin{aligned}
& T(x, y, t)=\frac{1}{\rho c(4 \pi K \tau)}\left[e^{-\left(r_{00}^{00}\right)^{2} / 4 K \tau}+\sum_{m=1}^{N S_{y}}(-1)^{(m-1)}\right. \\
& \cdot \sum_{j=1}^{4}(-1)^{(j !+1)} e^{-\left(r_{0 j}^{0 m}\right)^{2} / 4 K \tau}+\sum_{l=1}^{N S_{x}}(-1)^{(l-1)} \\
& \cdot \sum_{i=1}^{4}(-1)^{((i-1) !(i+1)+1)}\left[e^{-\left(r_{i 0}^{l 0}\right)^{2} / 4 K \tau}\left[\sum_{j=1}^{4}(-1)^{(j !+1)} e^{-\left(r_{i j}^{l m}\right)^{2} / 4 K \tau}\right]\right] \\
& \left.+\sum_{m=1}^{N S_{y}}(-1)^{(m-1)}\right] \\
& T(x, y, \omega)=\frac{-i}{4 \lambda}\left[H_{0}\left(k_{1} r_{00}^{00}\right)+\sum_{m=1}^{N S_{y}}(-1)^{(m-1)}\right.
\end{aligned}
$$

$$
\begin{aligned}
& \cdot \sum_{j=1}^{4}(-1)^{(j !+1)} H_{0}\left(k_{1} r_{0 j}^{0 m}\right)+\sum_{l=1}^{N S_{x}}(-1)^{(l-1)} \\
& \cdot \sum_{i=1}^{4}(-1)^{((i-1) !(i+1)+1)}\left[H_{0}\left(k_{1} r_{i 0}^{l 0}\right)\right. \\
& \left.\left.+\sum_{m=1}^{N S_{y}}(-1)^{(m-1)} \sum_{j=1}^{4}(-1)^{(j !+1)} H_{0}\left(k_{1} r_{i j}^{l m}\right)\right]\right] .
\end{aligned}
$$

Equations for the solid rectangular pipe (Cases 1-4), subjected to a $3 \mathrm{D}$ heat source, are as follows.

Case 1. Consider

$$
\begin{aligned}
& T(x, y, z, t)=\frac{1}{\rho c(4 \pi K \tau)^{1.5}}\left[e^{-\left(r_{000}^{000}\right)^{2} / 4 K \tau}+\sum_{m=1}^{N S_{y}} \sum_{j=1}^{4} e^{-\left(r_{0 j 0}^{0 m 0}\right)^{2} / 4 K \tau}+\sum_{l=1}^{N S_{x}} \sum_{i=1}^{4}\left[e^{-\left(r_{i 00}^{l 00}\right)^{2} / 4 K \tau}+\sum_{m=1}^{N S_{y}} \sum_{j=1}^{4} e^{-\left(r_{i j 0}^{l m 0}\right)^{2} / 4 K \tau}\right]\right], \\
& T(x, y, z, \omega)=\frac{1}{2 \lambda}\left[\frac{e^{-\sqrt{i \omega / K} r_{000}^{000}}}{r_{000}^{000}}+\sum_{m=1}^{N S_{y}} \sum_{j=1}^{4} \frac{e^{-\sqrt{i \omega / K} r_{0 j 0}^{0 m 0}}}{r_{0 j 0}^{0 m 0}}+\sum_{l=1}^{N S_{x}} \sum_{i=1}^{4}\left[\frac{e^{-\sqrt{i \omega / K} r_{i 00}^{l 00}}}{r_{i 00}^{l 00}}+\sum_{m=1}^{N S_{y}} \sum_{j=1}^{4} \frac{e^{-\sqrt{i \omega / K} r_{i j 0}^{l m 0}}}{r_{i j 0}^{l m 0}}\right]\right], \\
& T(x, y, z, \omega)=\frac{-i \pi}{2 \lambda L_{x}}\left[\sum_{m_{k}=-\infty}^{\infty}\left[H_{0}\left(k_{1 z^{\prime}} r_{00}^{00}\right) e^{-i k_{z}\left(\bar{z}_{0}\right)}\right]+\sum_{m=1}^{N S_{y}} \sum_{j=1}^{4} \sum_{m_{k}=-\infty}^{\infty}\left[H_{0}\left(k_{1 z} r_{0 j}^{0 m}\right) e^{-i k_{z}\left(\bar{z}_{0}\right)}\right]\right. \\
& \left.\quad+\sum_{l=1}^{N S_{x}} \sum_{i=1}^{4}\left[\sum_{m_{k}=-\infty}^{\infty}\left[H_{0}\left(k_{1 z} r_{i 0}^{l 0}\right) e^{-i k_{z}\left(\bar{z}_{0}\right)}\right]+\sum_{m=1}^{N S_{y}} \sum_{j=1}^{4} \sum_{m_{k}=-\infty}^{\infty}\left[H_{0}\left(k_{1 z} r_{i j}^{l m}\right) e^{-i k_{z}\left(\bar{z}_{0}\right)}\right]\right]\right]
\end{aligned}
$$

Case 2. Consider

$$
\begin{aligned}
& T(x, y, z, t)=\frac{1}{\rho c(4 \pi K \tau)^{1.5}}\left[e^{-\left(r_{000}^{000}\right)^{2} / 4 K \tau}\right. \\
& +\sum_{m=1}^{N S_{y}} \sum_{j=1}^{4}(-1)^{(j-1) !} e^{-\left(r_{0 j 0}^{000}\right)^{2} / 4 K \tau}+\sum_{l=1}^{N S_{x}} \sum_{i=1}^{4}(-1)^{(i-1) !} \\
& \left.\cdot\left[e^{-\left(r_{i 00}^{l 00}\right)^{2} / 4 K \tau}+\sum_{m=1}^{N S_{y}} \sum_{j=1}^{4}(-1)^{(j-1) !} e^{-\left(r_{i j 0}^{l m 0}\right)^{2} / 4 K \tau}\right]\right], \\
& T(x, y, z, \omega)=\frac{1}{2 \lambda}\left[\frac{e^{-\sqrt{i \omega / K} r_{000}^{000}}}{r_{000}^{000}}\right.
\end{aligned}
$$

as the sum of $2.5 \mathrm{D}$ sources.

$$
+\sum_{m=1}^{N S_{y}} \sum_{j=1}^{4}(-1)^{(j-1) !} \frac{e^{-\sqrt{i \omega / K} r_{0 j 0}^{0 m 0}}}{r_{0 j 0}^{0 m 0}}+\sum_{l=1}^{N S_{x}} \sum_{i=1}^{4}(-1)^{(i-1) !}
$$$$
\left.\cdot\left[\frac{e^{-\sqrt{i \omega / K} r_{i 00}^{l 00}}}{r_{i 00}^{l 00}}+\sum_{m=1}^{N S_{y}} \sum_{j=1}^{4}(-1)^{(j-1) !} \frac{e^{-\sqrt{i \omega / K} r_{i j 0}^{l m 0}}}{r_{i j 0}^{l m 0}}\right]\right],
$$$$
T(x, y, z, \omega)=\frac{-i \pi}{2 \lambda L_{x}}\left[\sum_{m_{k}=-\infty}^{\infty}\left[H_{0}\left(k_{1 z} r_{00}^{00}\right) e^{-i k_{z}\left(\bar{z}_{0}\right)}\right]\right.
$$$$
+\sum_{m=1}^{N S_{y}} \sum_{j=1}^{4}(-1)^{(j-1) !} \sum_{m_{k}=-\infty}^{\infty}\left[H_{0}\left(k_{1 z} r_{0 j}^{0 m}\right) e^{-i k_{z}\left(\bar{z}_{0}\right)}\right]
$$$$
+\sum_{l=1}^{N S_{x}} \sum_{i=1}^{4}(-1)^{(i-1) !}\left[\sum_{m_{k}=-\infty}^{\infty}\left[H_{0}\left(k_{1 z} r_{i 0}^{l 0}\right) e^{-i k_{z}\left(\bar{z}_{0}\right)}\right]\right.
$$ 


$$
\begin{aligned}
& +\sum_{m=1}^{N S_{y}} \sum_{j=1}^{4}(-1)^{(j-1) !} \\
& \left.\cdot \sum_{m_{k}=-\infty}^{\infty}\left[H_{0}\left(k_{1 z} r_{i j}^{l m}\right) e^{-i k_{z}\left(\bar{z}_{0}\right)}\right]\right]
\end{aligned}
$$

as the sum of $2.5 \mathrm{D}$ sources.

Case 3. Consider

$$
\begin{aligned}
& T(x, y, z, t)=\frac{1}{\rho c(4 \pi K \tau)^{1.5}}\left[e^{-\left(r_{000}^{000}\right)^{2} / 4 K \tau}\right. \\
& +\sum_{m=1}^{N S_{y}}(-1)^{(m-1)} \sum_{j=1}^{4}(-1)^{((j-1) !(j+1)+1)} e^{-\left(r_{0 j 0}^{0 m 0}\right)^{2} / 4 K \tau} \\
& +\sum_{l=1}^{N S_{x}}(-1)^{(l-1)} \sum_{i=1}^{4}(-1)^{(i !+1)}\left[e^{-\left(r_{i 00}^{l 00}\right)^{2} / 4 K \tau}\right. \\
& +\sum_{m=1}^{N S_{y}}(-1)^{(m-1)} \\
& \left.\left.\cdot \sum_{j=1}^{4}(-1)^{((j-1) !(j+1)+1)} e^{-\left(r_{i j 0}^{l m 0}\right)^{2} / 4 K \tau}\right]\right], \\
& T(x, y, z, \omega)=\frac{1}{2 \lambda}\left[\frac{e^{-\sqrt{i \omega / K} r_{000}^{000}}}{r_{000}^{000}}+\sum_{m=1}^{N S_{y}}(-1)^{(m-1)}\right. \\
& \cdot \sum_{j=1}^{4}(-1)^{((j-1) !(j+1)+1)} \frac{e^{-\sqrt{i \omega / K} r_{0 j 0}^{0 m 0}}}{r_{0 j 0}^{0 m 0}}+\sum_{l=1}^{N S_{x}}(-1)^{(l-1)} \\
& \cdot \sum_{i=1}^{4}(-1)^{(i !-1)}\left[\frac{e^{-\sqrt{i \omega / K} r_{i 00}^{l 00}}}{r_{i 00}^{l 00}}+\sum_{m=1}^{N S_{y}}(-1)^{(m-1)}\right. \\
& \left.\left.\cdot \sum_{j=1}^{4}(-1)^{((j-1) !(j+1)+1)} \frac{e^{-\sqrt{i \omega / K} r_{i j 0}^{l m 0}}}{r_{i j 0}^{l m 0}}\right]\right] \text {, } \\
& T(x, y, z, \omega)=\frac{-i \pi}{2 \lambda L_{x}}\left[\sum_{m_{k}=-\infty}^{\infty}\left[H_{0}\left(k_{1 z} r_{00}^{00}\right) e^{-i k_{z}\left(\bar{z}_{0}\right)}\right]\right. \\
& +\sum_{m=1}^{N S_{y}}(-1)^{(m-1)} \sum_{j=1}^{4}(-1)^{((j-1) !(j+1)+1)} \\
& \sum_{m_{k}=-\infty}^{\infty}\left[H_{0}\left(k_{1 z} r_{0 j}^{0 m}\right) e^{-i k_{z}\left(\bar{z}_{0}\right)}\right]+\sum_{l=1}^{N S_{x}}(-1)^{(l-1)} \\
& T(x, y, z, t)=\frac{1}{\rho c(4 \pi K \tau)^{1.5}}\left[e^{-\left(r_{000}^{000}\right)^{2} / 4 K \tau}\right. \\
& +\sum_{m=1}^{N S_{y}}(-1)^{(m-1)} \sum_{j=1}^{4}(-1)^{(j !+1)} e^{-\left(r_{0 j 0}^{0 m 0}\right)^{2} / 4 K \tau} \\
& +\sum_{l=1}^{N S_{x}}(-1)^{(l-1)} \sum_{i=1}^{4}(-1)^{((i-1) !(i+1)+1)}\left[e^{-\left(r_{i 00}^{l 00}\right)^{2} / 4 K \tau}\right. \\
& \left.\left.+\sum_{m=1}^{N S_{y}}(-1)^{(m-1)} \sum_{j=1}^{4}(-1)^{(j !+1)} e^{-\left(r_{i j 0}^{l m 0}\right)^{2} / 4 K \tau}\right]\right] \\
& T(x, y, z, \omega)=\frac{1}{2 \lambda}\left[\frac{e^{-\sqrt{i \omega / K} r_{000}^{000}}}{r_{000}^{000}}\right. \\
& +\sum_{m=1}^{N S_{y}}\left[(-1)^{(m-1)} \sum_{j=1}^{4}(-1)^{(j !+1)} \frac{e^{-\sqrt{i \omega / K} r_{0 j 0}^{0 m 0}}}{r_{0 j 0}^{0 m 0}}\right] \\
& +\sum_{l=1}^{N S_{x}}(-1)^{(l-1)} \sum_{i=1}^{4}(-1)^{((j-1) !(j+1)+1)}\left[\frac{e^{-\sqrt{i \omega / K} r_{i 00}^{l 00}}}{r_{i 00}^{l 00}}\right. \\
& \left.\left.+\sum_{m=1}^{N S_{y}}(-1)^{(m-1)} \sum_{j=1}^{4}(-1)^{(j !+1)} \frac{e^{-\sqrt{i \omega / K} r_{i j 0}^{l m 0}}}{r_{i j 0}^{l m 0}}\right]\right] \\
& T(x, y, z, \omega)=\frac{-i \pi}{2 \lambda L_{x}}\left[\sum_{m_{k}=-\infty}^{\infty}\left[H_{0}\left(k_{1 z} r_{00}^{00}\right) e^{-i k_{z}\left(\bar{z}_{0}\right)}\right]\right. \\
& +\sum_{m=1}^{N S_{y}}(-1)^{(m-1)} \sum_{j=1}^{4}(-1)^{(j !+1)} \\
& \cdot \sum_{m_{k}=-\infty}^{\infty}\left[H_{0}\left(k_{1 z^{2}} r_{0 j}^{0 m}\right) e^{-i k_{z}\left(\bar{z}_{0}\right)}\right]+\sum_{l=1}^{N S_{x}}(-1)^{(l-1)}
\end{aligned}
$$

$$
\begin{aligned}
& \cdot \sum_{i=1}^{4}(-1)^{(i !-1)}\left[\sum_{m_{k}=-\infty}^{\infty}\left[H_{0}\left(k_{1 z} r_{i 0}^{l 0}\right) e^{-i k_{z}\left(\bar{z}_{0}\right)}\right]\right. \\
& +\sum_{m=1}^{N S_{y}}(-1)^{(m-1)} \sum_{j=1}^{4}(-1)^{((j-1) !(j+1)+1)} \\
& \left.\cdot \sum_{m_{k}=-\infty}^{\infty}\left[H_{0}\left(k_{1 z^{r}} r_{i j}^{l m}\right) e^{-i k_{z}\left(\bar{z}_{0}\right)}\right]\right]
\end{aligned}
$$

as the sum of $2.5 \mathrm{D}$ sources. 


$$
\begin{aligned}
& \cdot \sum_{i=1}^{4}(-1)^{((i-1) !(j+1)+1)}\left[\sum_{m_{k}=-\infty}^{\infty}\left[H_{0}\left(k_{1 z} r_{i 0}^{l 0}\right) e^{-i k_{z}\left(\bar{z}_{0}\right)}\right]\right. \\
& +\sum_{m=1}^{N S_{y}}(-1)^{(m-1)} \sum_{j=1}^{4}(-1)^{(j !+1)} \\
& \left.\left.\cdot \sum_{m_{k}=-\infty}^{\infty}\left[H_{0}\left(k_{1 z} r_{i j}^{l m}\right) e^{-i k_{z}\left(\bar{z}_{0}\right)}\right]\right]\right]
\end{aligned}
$$

\subsection{Three-Dimensional Space}

(a) Solid Open Box Defined by $y \geq 0, y \leq d_{2}, x \geq 0, x \leq$ $d_{1}$, and $z \leq d_{3}$. Boundary conditions prescribed for the solid open box (Cases 1-4) are shown in Figure 15.

Equations for the solid open box (Cases 1-4), subjected to a $3 \mathrm{D}$ heat source, are as follows.

Case 1. Consider

as the sum of $2.5 \mathrm{D}$ sources.

(46)

$$
\begin{aligned}
& T(x, y, z, t)=\frac{1}{\rho c(4 \pi K \tau)^{1.5}} \sum_{n=0}^{1}\left[e^{-\left(r_{00 n}^{00 n}\right)^{2} / 4 K \tau}+\sum_{m=1}^{N S_{y}} \sum_{j=1}^{4} e^{-\left(r_{0 j n}^{0 m n}\right)^{2} / 4 K \tau}+\sum_{l=1}^{N S_{x}} \sum_{i=1}^{4}\left[e^{-\left(r_{i 0 n}^{l 0 n}\right)^{2} / 4 K \tau}+\sum_{m=1}^{N S_{y}} \sum_{j=1}^{4} e^{-\left(r_{i j n}^{l m n}\right)^{2} / 4 K \tau}\right]\right], \\
& T(x, y, z, \omega)=\frac{1}{2 \lambda} \sum_{n=0}^{1}\left[\frac{e^{-\sqrt{i \omega / K} r_{00 n}^{00 n}}}{r_{00 n}^{00 n}}+\sum_{m=1}^{N S_{y}} \sum_{j=1}^{4} \frac{e^{-\sqrt{i \omega / K} r_{0 j n}^{0 m n}}}{r_{0 j n}^{0 m n}}+\sum_{l=1}^{N S_{x}} \sum_{i=1}^{4}\left[\frac{e^{-\sqrt{i \omega / K} r_{i 0 n}^{l 0 n}}}{r_{i 0 n}^{l 0 n}}+\sum_{m=1}^{N S_{y}} \sum_{j=1}^{4} \frac{e^{-\sqrt{i \omega / K} r_{i j n}^{l m n}}}{r_{i j n}^{l m n}}\right]\right], \\
& T(x, y, z, \omega)=\frac{-i \pi}{2 \lambda L_{x}} \sum_{n=0}^{1}\left[\sum_{m_{k}=-\infty}^{\infty}\left[H_{0}\left(k_{1 z} r_{00}^{00}\right) e^{-i k_{z}\left(\bar{z}_{n}\right)}\right]+\sum_{m=1}^{N S_{y}} \sum_{j=1}^{4} \sum_{m_{k}=-\infty}^{\infty}\left[H_{0}\left(k_{1 z} r_{0 j}^{0 m}\right) e^{-i k_{z}\left(\bar{z}_{n}\right)}\right]\right. \\
& \left.\quad+\sum_{l=1}^{N S_{x}} \sum_{i=1}^{4}\left[\sum_{m_{k}=-\infty}^{\infty}\left[H_{0}\left(k_{1 z^{2}} r_{i 0}^{l 0}\right) e^{-i k_{z}\left(\bar{z}_{n}\right)}\right]+\sum_{m=1}^{N S_{y}} \sum_{j=1}^{4} \sum_{m_{k}=-\infty}^{\infty}\left[H_{0}\left(k_{1 z} r_{i j}^{l m}\right) e^{-i k_{z}\left(\bar{z}_{n}\right)}\right]\right]\right]
\end{aligned}
$$

Case 2. Consider

$$
\begin{array}{r}
T(x, y, z, t)=\frac{1}{\rho c(4 \pi K \tau)^{1.5}} \sum_{n=0}^{1}(-1)^{n}\left[e^{-\left(r_{00 n}^{00 n}\right)^{2} / 4 K \tau}\right. \\
+\sum_{m=1}^{N S_{y}} \sum_{j=1}^{4}(-1)^{(j-1) !} e^{-\left(r_{0 j n}^{(0 m n}\right)^{2} / 4 K \tau}+\sum_{l=1}^{N S_{x}} \sum_{i=1}^{4}(-1)^{(i-1) !} \\
\left.\cdot\left[e^{-\left(r_{i 0 n}^{l 0 n}\right)^{2} / 4 K \tau}+\sum_{m=1}^{N S_{y}} \sum_{j=1}^{4}(-1)^{(j-1) !} e^{-\left(r_{i j n}^{l m n}\right)^{2} / 4 K \tau}\right]\right], \\
T(x, y, z, \omega)=\frac{1}{2 \lambda} \sum_{n=0}^{1}(-1)^{n}\left[\frac{e^{-\sqrt{i \omega / K} r_{00 n}^{00 n}}}{r_{00 n}^{00 n}}\right. \\
+\sum_{m=1}^{N S_{y}} \sum_{j=1}^{4}(-1)^{(j-1) !} \frac{e^{-\sqrt{i \omega / K} r_{0 j n}^{0 m n}}}{r_{0 j n}^{0 m n}}+\sum_{l=1}^{N S_{x}} \sum_{i=1}^{4}(-1)^{(i-1) !} \\
\left.\cdot\left[\frac{e^{-\sqrt{i \omega / K} r_{i 0 n}^{l 0 n}}}{r_{i 0 n}^{l 0 n}}+\sum_{m=1}^{N S_{y}} \sum_{j=1}^{4}(-1)^{(j-1) !} \frac{e^{-\sqrt{i \omega / K} r_{i j n}^{l m n}}}{r_{i j n}^{l m n}}\right]\right],
\end{array}
$$

$$
T(x, y, z, \omega)=\frac{-i \pi}{2 \lambda L_{x}} \sum_{n=0}^{1}(-1)^{n}
$$

$\left[\sum_{m_{k}=-\infty}^{\infty}\left[H_{0}\left(k_{1 z} r_{00}^{00}\right) e^{-i k_{z}\left(\bar{z}_{n}\right)}\right]\right.$

$+\sum_{m=1}^{N S_{y}} \sum_{j=1}^{4}(-1)^{(j-1) !} \sum_{m_{k}=-\infty}^{\infty}\left[H_{0}\left(k_{1 z} r_{0 j}^{0 m}\right) e^{-i k_{z}\left(\bar{z}_{n}\right)}\right]$

$+\sum_{l=1}^{N S_{x}} \sum_{i=1}^{4}(-1)^{(i-1) !}\left[\sum_{m_{k}=-\infty}^{\infty}\left[H_{0}\left(k_{1 z} r_{i 0}^{l 0}\right) e^{-i k_{z}\left(\bar{z}_{n}\right)}\right]\right.$

$+\sum_{m=1}^{N S_{y}} \sum_{j=1}^{4}(-1)^{(j-1) !}$

$\left.\left.\cdot \sum_{m_{k}=-\infty}^{\infty}\left[H_{0}\left(k_{1 z} r_{i j}^{l m}\right) e^{-i k_{z}\left(\bar{z}_{n}\right)}\right]\right]\right]$

as the sum of $2.5 \mathrm{D}$ sources. 
Case 3. Consider

$$
\begin{aligned}
& T(x, y, z, t)=\frac{1}{\rho c(4 \pi K \tau)^{1.5}} \sum_{n=0}^{1}\left[e^{-\left(r_{00 n}^{00 n}\right)^{2} / 4 K \tau}\right. \\
& +\sum_{m=1}^{N S_{y}}(-1)^{(m-1)} \sum_{j=1}^{4}(-1)^{((j-1) !(j+1)+1)} e^{-\left(r_{0 j n}^{0 m n}\right)^{2} / 4 K \tau} \\
& +\sum_{l=1}^{N S_{x}}(-1)^{(l-1)} \sum_{i=1}^{4}(-1)^{(i !+1)}\left[e^{-\left(r_{i 0 n}^{l 0 n}\right)^{2} / 4 K \tau}\right. \\
& +\sum_{m=1}^{N S_{y}}(-1)^{(m-1)} \\
& \left.\left.\cdot \sum_{j=1}^{4}(-1)^{((j-1) !(j+1)+1)} e^{-\left(r_{i j n}^{l m n}\right)^{2} / 4 K \tau}\right]\right] \text {, } \\
& T(x, y, z, \omega)=\frac{1}{2 \lambda} \sum_{n=0}^{1}\left[\frac{e^{-\sqrt{i \omega / K} r_{00 n}^{00 n}}}{r_{000}^{000}}+\sum_{m=1}^{N S_{y}}(-1)^{(m-1)}\right. \\
& \cdot \sum_{j=1}^{4}(-1)^{((j-1) !(j+1)+1)} \frac{e^{-\sqrt{i \omega / K} r_{0 j n}^{0 m n}}}{r_{0 j n}^{0 m n}}+\sum_{l=1}^{N S_{x}}(-1)^{(l-1)} \\
& \cdot \sum_{i=1}^{4}(-1)^{(i !-1)}\left[\frac{e^{-\sqrt{i \omega / K} r_{i 0 n}^{l 0 n}}}{r_{i 0 n}^{l 0 n}}+\sum_{m=1}^{N S_{y}}(-1)^{(m-1)}\right. \\
& \left.\left.\cdot \sum_{j=1}^{4}(-1)^{((j-1) !(j+1)+1)} \frac{e^{-\sqrt{i \omega / K} r_{i j 0}^{l m n}}}{r_{i j n}^{l m n}}\right]\right] \text {, } \\
& T(x, y, z, \omega)=\frac{-i \pi}{2 \lambda L_{x}}\left[\sum_{m_{k}=-\infty}^{\infty}\left[H_{0}\left(k_{1 z} r_{00}^{00}\right) e^{-i k_{z}\left(\bar{z}_{n}\right)}\right]\right. \\
& +\sum_{m=1}^{N S_{y}}(-1)^{(m-1)} \sum_{j=1}^{4}(-1)^{((j-1) !(j+1)+1)} \\
& \cdot \sum_{m_{k}=-\infty}^{\infty}\left[H_{0}\left(k_{1 z} r_{0 j}^{0 m}\right) e^{-i k_{z}\left(\bar{z}_{n}\right)}\right]+\sum_{l=1}^{N S_{x}}(-1)^{(l-1)} \\
& \cdot \sum_{i=1}^{4}(-1)^{(i !-1)}\left[\sum_{m_{k}=-\infty}^{\infty}\left[H_{0}\left(k_{1 z} r_{i 0}^{l 0}\right) e^{-i k_{z}\left(\bar{z}_{n}\right)}\right]\right. \\
& +\sum_{m=1}^{N S_{y}}(-1)^{(m-1)} \sum_{j=1}^{4}(-1)^{((j-1) !(j+1)+1)} \\
& \left.\left.\cdot \sum_{m_{k}=-\infty}^{\infty}\left[H_{0}\left(k_{1 z} r_{i j}^{l m}\right) e^{-i k_{z}\left(\bar{z}_{n}\right)}\right]\right]\right]
\end{aligned}
$$

as the sum of $2.5 \mathrm{D}$.
Case 4. Consider

$$
\begin{aligned}
& T(x, y, z, t)=\frac{1}{\rho c(4 \pi K \tau)^{1.5}} \sum_{n=0}^{1}(-1)^{n}\left[e^{-\left(r_{00 n}^{00 n}\right)^{2} / 4 K \tau}\right. \\
& +\sum_{m=1}^{N S_{y}}(-1)^{(m-1)} \sum_{j=1}^{4}(-1)^{(j !+1)} e^{-\left(r_{0 j n}^{0 m n}\right)^{2} / 4 K \tau} \\
& +\sum_{l=1}^{N S_{x}}(-1)^{(l-1)} \sum_{i=1}^{4}(-1)^{((i-1) !(i+1)+1)}\left[e^{-\left(r_{i 0 n}^{l 0 n}\right)^{2} / 4 K \tau}\right. \\
& \left.\left.+\sum_{m=1}^{N S_{y}}(-1)^{(m-1)} \sum_{j=1}^{4}(-1)^{(j !+1)} e^{-\left(r_{i j n}^{l m n}\right)^{2} / 4 K \tau}\right]\right] \text {, } \\
& T(x, y, z, \omega)=\frac{1}{2 \lambda} \sum_{n=0}^{1}(-1)^{n}\left[\frac{e^{-\sqrt{i \omega / K} r_{00 n}^{00 n}}}{r_{00 n}^{00 n}}\right. \\
& +\sum_{m=1}^{N S_{y}}(-1)^{(m-1)} \sum_{j=1}^{4}(-1)^{(j !+1)} \frac{e^{-\sqrt{i \omega / K} r_{0 j n}^{0 m n}}}{r_{0 j n}^{0 m n}} \\
& +\sum_{l=1}^{N S_{x}}(-1)^{(l-1)} \sum_{i=1}^{4}(-1)^{((i-1) !(i+1)+1)}\left[\frac{e^{-\sqrt{i \omega / K} r_{i 0 n}^{l 0 n}}}{r_{i 0 n}^{l 0 n}}\right. \\
& \left.\left.+\sum_{m=1}^{N S_{y}}(-1)^{(m-1)} \sum_{j=1}^{4}(-1)^{(j !+1)} \frac{e^{-\sqrt{i \omega / K} r_{i j n}^{l m n}}}{r_{i j n}^{l m n}}\right]\right], \\
& T(x, y, z, \omega)=\frac{-i \pi}{2 \lambda L_{x}} \sum_{n=0}^{1}(-1)^{n} \\
& \cdot\left[\sum_{m_{k}=-\infty}^{\infty}\left[H_{0}\left(k_{1 z} r_{00}^{00}\right) e^{-i k_{z}\left(\bar{z}_{n}\right)}\right]+\sum_{m=1}^{N S_{y}}(-1)^{(m-1)}\right. \\
& \cdot \sum_{j=1}^{4}(-1)^{(j !+1)} \sum_{m_{k}=-\infty}^{\infty}\left[H_{0}\left(k_{1 z} r_{0 j}^{0 m}\right) e^{-i k_{z}\left(\bar{z}_{n}\right)}\right] \\
& +\sum_{l=1}^{N S_{x}}(-1)^{(l-1)} \sum_{i=1}^{4}(-1)^{((i-1) !(i+1)+1)} \\
& \cdot\left[\sum_{m_{k}=-\infty}^{\infty}\left[H_{0}\left(k_{1 z} r_{i 0}^{l 0}\right) e^{-i k_{z}\left(\bar{z}_{n}\right)}\right]+\sum_{m=1}^{N S_{y}}(-1)^{(m-1) !}\right. \\
& \left.\left.\cdot \sum_{j=1}^{4}(-1)^{(j !+1)} \sum_{m_{k}=-\infty}^{\infty}\left[H_{0}\left(k_{1 z} r_{i j}^{l m}\right) e^{-i k_{z}\left(\bar{z}_{n}\right)}\right]\right]\right]
\end{aligned}
$$

as the sum of $2.5 \mathrm{D}$ sorces.

(b) 3D Parallelepiped Box Defined by $y \geq 0, y \leq d_{2}, x \geq 0$, $x \leq d_{1}, z \geq 0$, and $z \leq d_{3}$. Boundary conditions for the 3D parallelepiped box (Cases 1-4) are shown in Figure 16. 
Equations for the 3D parallelepiped box (Cases 1- Case 1. Consider 4), subjected to a 3D heat source, are as follows.

$$
\begin{aligned}
& T(x, y, z, t)=\frac{1}{\rho c(4 \pi K \tau)^{1.5}}\left[e^{-\left(r_{000}^{000}\right)^{2} / 4 K \tau}+\sum_{m=1}^{N S_{y}} \sum_{j=1}^{4} e^{-\left(r_{0 j 0}^{000}\right)^{2} / 4 K \tau}+\sum_{l=1}^{N S_{x}} \sum_{i=1}^{4}\left[e^{-\left(r_{i 00}^{l 00}\right)^{2} / 4 K \tau}+\sum_{m=1}^{N S_{y}} \sum_{j=1}^{4} e^{-\left(r_{i j j}^{m 0}\right)^{2} / 4 K \tau}\right]\right. \\
& \left.+\sum_{n=1}^{N S_{z}} \sum_{k=1}^{4}\left[e^{-\left(r_{00 k}^{(00 n}\right)^{2} / 4 K \tau}+\sum_{m=1}^{N S_{y}} \sum_{j=1}^{4} e^{-\left(r_{0 j k}^{0 m n}\right)^{2} / 4 K \tau}+\sum_{l=1}^{N S_{x}} \sum_{i=1}^{4}\left[e^{-\left(r_{i 0 k}^{(0 n)}\right)^{2} / 4 K \tau}+\sum_{m=1}^{N S_{y}} \sum_{j=1}^{4} e^{-\left(r_{i j k}^{l m n}\right)^{2} / 4 K \tau}\right]\right]\right] \\
& T(x, y, z, \omega)=\frac{1}{2 \lambda}\left[\frac{e^{-\sqrt{i \omega / K} r_{000}^{000}}}{r_{000}^{000}}+\sum_{m=1}^{N S_{y}} \sum_{j=1}^{4} \frac{e^{-\sqrt{i \omega / K} r_{0 j 0}^{0 m 0}}}{r_{0 j 0}^{0 m 0}}+\sum_{l=1}^{N S_{x}} \sum_{i=1}^{4}\left[\frac{e^{-\sqrt{i \omega / K} r_{i 00}^{r 00}}}{r_{i 00}^{l 00}}+\sum_{m=1}^{N S_{y}} \sum_{j=1}^{4} \frac{e^{-\sqrt{i \omega / K} r_{i j 0}^{l m 0}}}{r_{i j 0}^{l m 0}}\right]\right. \\
& +\sum_{n=1}^{N S_{z}} \sum_{k=1}^{4}\left[\frac{e^{-\sqrt{\omega \omega / K} r_{00 k}^{00 n}}}{r_{00 k}^{00 n}}+\sum_{m=1}^{N S_{y}} \sum_{j=1}^{4} \frac{e^{-\sqrt{i \omega / K} r_{0 j k}^{0 n m}}}{r_{0 j k}^{0 m n}}+\sum_{l=1}^{N S_{x}} \sum_{i=1}^{4}\left[\frac{e^{-\sqrt{i \omega / K} r_{i 0 k}^{l 0 n}}}{r_{i 0 k}^{l 0 n}}+\sum_{m=1}^{N S_{y}} \sum_{j=1}^{4} \frac{e^{-\sqrt{i \omega / K} r_{i j k}^{l m n}}}{r_{i j k}^{l m n}}\right]\right], \\
& T(x, y, z, \omega)=\frac{-i \pi}{2 \lambda L_{x}}\left[\sum_{m_{k}=-\infty}^{\infty}\left[H_{0}\left(k_{1 z} r_{00}^{00}\right) e^{-i k_{z}\left(\bar{z}_{0}\right)}\right]+\sum_{m=1}^{N S_{y}} \sum_{j=1}^{4} \sum_{m_{k}=-\infty}^{\infty}\left[H_{0}\left(k_{1 z} r_{0 j}^{0 m}\right) e^{-i k_{z}\left(\bar{z}_{0}\right)}\right]\right. \\
& +\sum_{l=1}^{N S_{x}} \sum_{i=1}^{4}\left[\sum_{m_{k}=-\infty}^{\infty}\left[H_{0}\left(k_{1 z} r_{i 0}^{l 0}\right) e^{-i k_{z}\left(\bar{z}_{0}\right)}\right]+\sum_{m=1}^{N S_{y}} \sum_{j=1}^{4} \sum_{m_{k}=-\infty}^{\infty}\left[H_{0}\left(k_{1 z} r_{i j}^{l m}\right) e^{-i k_{z}\left(\bar{z}_{0}\right)}\right]\right] \\
& \left.+\sum_{n=1}^{N S_{z}} \sum_{k=1}^{4}\left[\sum_{m_{k}=-\infty}^{\infty}\left[H_{0}\left(k_{1 z} r_{00}^{00}\right) e^{-i k_{z}\left(\bar{z}_{n}\right)}\right]+\sum_{m=1}^{N S_{y}} \sum_{j=1}^{4} \sum_{m_{k}=-\infty}^{\infty}\left[H_{0}\left(k_{1 z} r_{0 j}^{0 m}\right) e^{-i k_{z}\left(\bar{z}_{n}\right)}\right]+\sum_{l=1}^{N S_{x}} \sum_{i=1}^{4}\left[\sum_{m_{k}=-\infty}^{\infty}\left[H_{0}\left(k_{1 z} r_{i 0}^{l 0}\right) e^{-i k_{z}\left(\bar{z}_{n}\right)}\right]+\sum_{m=1}^{N S_{y}} \sum_{j=1}^{4} \sum_{m_{k}=-\infty}^{\infty}\left[H_{0}\left(k_{1 z} r_{i j}^{l m}\right) e^{-i k_{z}\left(\bar{z}_{n}\right)}\right]\right]\right]\right]
\end{aligned}
$$

Case 2. Consider

$$
\begin{aligned}
T( & x, y, z, t)=\frac{1}{\rho c(4 \pi K \tau)^{1.5}}\left[e^{-\left(r_{000}^{000}\right)^{2} / 4 K \tau}\right. \\
+ & \sum_{m=1}^{N S_{y}} \sum_{j=1}^{4}(-1)^{(j-1) !} e^{-\left(r_{0 j 0}^{000}\right)^{2} / 4 K \tau}+\sum_{l=1}^{N S_{x}} \sum_{i=1}^{4}(-1)^{(i-1) !} \\
& \cdot\left[e^{-\left(r_{i 00}^{l 00}\right)^{2} / 4 K \tau}+\sum_{m=1}^{N S_{y}} \sum_{j=1}^{4}(-1)^{(j-1) !} e^{-\left(r_{i j 0}^{l m 0}\right)^{2} / 4 K \tau}\right] \\
+ & \sum_{n=1}^{N S_{z}} \sum_{k=1}^{4}(-1)^{(k-1) !}\left[e^{-\left(r_{00 k}^{00 n}\right)^{2} / 4 K \tau}\right] \\
& +\sum_{m=1}^{N S_{y}} \sum_{j=1}^{4}(-1)^{(j-1) !} e^{-\left(r_{0 j k}^{0 m n}\right)^{2} / 4 K \tau}+\sum_{l=1}^{N S_{x}} \sum_{i=1}^{4}(-1)^{(i-1) !} \\
T(x, y, z, \omega)=\frac{1}{2 \lambda}\left[\frac{e^{-\sqrt{i \omega / K} r_{000}^{000}}}{r_{000}^{000}}\right. & {\left.\left[e^{-\left(r_{i 0 k}^{l 0 n}\right)^{2} / 4 K \tau}+\sum_{m=1}^{N S_{y}} \sum_{j=1}^{4}(-1)^{(j-1) !} e^{-\left(r_{i j k}^{l m n}\right)^{2} / 4 K \tau}\right]\right] }
\end{aligned}
$$$$
+\sum_{m=1}^{N S_{y}} \sum_{j=1}^{4}(-1)^{(j-1) !} \frac{e^{-\sqrt{i \omega / K} r_{0 j 0}^{0 m 0}}}{r_{0 j 0}^{0 m 0}}+\sum_{l=1}^{N S_{x}} \sum_{i=1}^{4}(-1)^{(i-1) !}
$$$$
\cdot\left[\frac{e^{-\sqrt{i \omega / K} r_{i 00}^{100}}}{r_{i 00}^{l 00}}+\sum_{m=1}^{N S_{y}} \sum_{j=1}^{4}(-1)^{(j-1) !} \frac{e^{-\sqrt{i \omega / K} r_{i j 0}^{l m 0}}}{r_{i j 0}^{l m 0}}\right]
$$$$
+\sum_{n=1}^{N S_{z}} \sum_{k=1}^{4}(-1)^{(k-1) !}\left[\frac{e^{-\sqrt{i \omega / K} r_{00 k}^{00 n}}}{r_{00 k}^{00 n}}\right.
$$$$
+\sum_{m=1}^{N S_{y}} \sum_{j=1}^{4}(-1)^{(j-1) !} \frac{e^{-\sqrt{i \omega / K} r_{0 j k}^{0 m n}}}{r_{0 j k}^{0 m n}}+\sum_{l=1}^{N S_{x}} \sum_{i=1}^{4}(-1)^{(i-1) !}
$$$$
\left.\cdot\left[\frac{e^{-\sqrt{i \omega / K} r_{i 0 k}^{l 0 n}}}{r_{i 0 k}^{l 0 n}}+\sum_{m=1}^{N S_{y}} \sum_{j=1}^{4}(-1)^{(j-1) !} \frac{e^{-\sqrt{i \omega / K} r_{i j k}^{l m n}}}{r_{i j k}^{l m n}}\right]\right],
$$$$
T(x, y, z, \omega)=\frac{-i \pi}{2 \lambda L_{x}}\left[\sum_{m_{k}=-\infty}^{\infty}\left[H_{0}\left(k_{1 z} r_{00}^{00}\right) e^{-i k_{z}\left(\bar{z}_{0}\right)}\right]\right.
$$$$
+\sum_{m=1}^{N S_{y}} \sum_{j=1}^{4}(-1)^{(j-1) !} \sum_{m_{k}=-\infty}^{\infty}\left[H_{0}\left(k_{1 z} r_{0 j}^{0 m}\right) e^{-i k_{z}\left(\bar{z}_{0}\right)}\right]
$$$$
+\sum_{l=1}^{N S_{x}} \sum_{i=1}^{4}(-1)^{(i-1) !}\left[\sum_{m_{k}=-\infty}^{\infty}\left[H_{0}\left(k_{1 z} r_{i 0}^{l 0}\right) e^{-i k_{z}\left(\bar{z}_{0}\right)}\right]\right.
$$$$
+\sum_{m=1}^{N S_{y}} \sum_{j=1}^{4}(-1)^{(j-1) !} \sum_{m_{k}=-\infty}^{\infty}\left[H_{0}\left(k_{1 z} r_{i j}^{l m}\right) e^{-i k_{z}\left(\bar{z}_{0}\right)}\right]
$$ 


$$
\begin{array}{lr}
+\sum_{n=1}^{N S_{z}} \sum_{k=1}^{4}(-1)^{(k-1) !}\left[\sum_{m_{k}=-\infty}^{\infty}\left[H_{0}\left(k_{1 z} r_{00}^{00}\right) e^{-i k_{z}\left(\bar{z}_{n}\right)}\right]\right. & \left.\left.+\sum_{m=1}^{N S_{y}} \sum_{j=1}^{4}(-1)^{(j-1) !} \sum_{m_{k}=-\infty}^{\infty}\left[H_{0}\left(k_{1 z} r_{i j}^{l m}\right) e^{-i k_{z}\left(\bar{z}_{n}\right)}\right]\right]\right] \\
+\sum_{m=1}^{N S_{y}} \sum_{j=1}^{4}(-1)^{(j-1) !} \sum_{m_{k}=-\infty}^{\infty}\left[H_{0}\left(k_{1 z r_{0 j}^{0 m}}\right) e^{-i k_{z}\left(\bar{z}_{n}\right)}\right] & \text { as the sum of 2.5D sources. }
\end{array}
$$$$
+\sum_{l=1}^{N S_{x}} \sum_{i=1}^{4}(-1)^{(i-1) !}\left[\sum_{m_{k}=-\infty}^{\infty}\left[H_{0}\left(k_{1 z} r_{i 0}^{l 0}\right) e^{-i k_{z}\left(\bar{z}_{n}\right)}\right]\right.
$$

$$
\begin{aligned}
& T(x, y, z, t)=\frac{1}{\rho c(4 \pi K \tau)^{1.5}}\left[e^{-\left(r_{000}^{000}\right)^{2} / 4 K \tau}+\sum_{m=1}^{N S_{y}}(-1)^{(m-1)} \sum_{j=1}^{4}(-1)^{((j-1) !(j+1)+1)} e^{-\left(r_{0 j 0}^{0 m 0}\right)^{2} / 4 K \tau}+\sum_{l=1}^{N S_{x}}(-1)^{(l-1)} \sum_{i=1}^{4}(-1)^{(i !+1)}\right. \\
& \cdot\left[e^{-\left(r_{i 00}^{l 00}\right)^{2} / 4 K \tau}+\sum_{m=1}^{N S_{y}}(-1)^{(m-1)} \sum_{j=1}^{4}(-1)^{((j-1) !(j+1)+1)} e^{-\left(r_{i j 0}^{l m 0}\right)^{2} / 4 K \tau}\right]+\sum_{n=1}^{N S_{z}}(-1)^{(n-1)} \sum_{k=1}^{4}(-1)^{(k !+1)}\left[e^{-\left(r_{00 k}^{00 n}\right)^{2} / 4 K \tau}\right. \\
& +\sum_{m=1}^{N S_{y}}(-1)^{(m-1)} \sum_{j=1}^{4}(-1)^{((j-1) !(j+1)+1)} e^{-\left(r_{0 j k}^{0 m n}\right)^{2} / 4 K \tau}+\sum_{l=1}^{N S_{x}}(-1)^{(l-1)} \sum_{i=1}^{4}(-1)^{(i !+1)} \\
& \left.\left.\cdot\left[e^{-\left(r_{i 0 k}^{l 0 n}\right)^{2} / 4 K \tau}+\sum_{m=1}^{N S_{y}}(-1)^{(m-1)} \sum_{j=1}^{4}(-1)^{((j-1) !(j+1)+1)} e^{-\left(r_{i j k}^{l m n}\right)^{2} / 4 K \tau}\right]\right]\right] \\
& T(x, y, z, \omega)=\frac{1}{2 \lambda}\left[\frac{e^{-\sqrt{i \omega / K} r_{000}^{000}}}{r_{000}^{000}}+\sum_{m=1}^{N S_{y}}\left[(-1)^{(m-1)} \sum_{j=1}^{4}(-1)^{((j-1) !(j+1)+1)} \frac{e^{-\sqrt{i \omega / K} r_{0 j 0}^{0 m 0}}}{r_{0 j 0}^{0 m 0}}\right]+\sum_{l=1}^{N S_{x}}(-1)^{(l-1)} \sum_{i=1}^{4}(-1)^{(i !+1)}\right. \\
& \cdot\left[\frac{e^{-\sqrt{i \omega / K} r_{i 00}^{l 00}}}{r_{i 00}^{l 00}}+\sum_{m=1}^{N S_{y}}(-1)^{(m-1)} \sum_{j=1}^{4}(-1)^{((j-1) !(j+1)+1)} \frac{e^{-\sqrt{i \omega / K} r_{i j 0}^{l m 0}}}{r_{i j 0}^{l m 0}}\right]+\sum_{n=1}^{N S_{z}}(-1)^{(n-1)} \sum_{k=1}^{4}(-1)^{(k !+1)}\left[\frac{e^{-\sqrt{i \omega / K} r_{00 k}^{00 n}}}{r_{00 k}^{00 n}}\right. \\
& +\sum_{m=1}^{N S_{y}}\left[(-1)^{(m-1)} \sum_{j=1}^{4}(-1)^{((j-1) !(j+1)+1)} \frac{e^{-\sqrt{i \omega / K} r_{0 j k}^{0 m n}}}{r_{0 j k}^{0 m n}}\right]+\sum_{l=1}^{N S_{x}}(-1)^{(l-1)} \sum_{i=1}^{4}(-1)^{(i !+1)} \\
& \left.\left[\frac{e^{-\sqrt{i \omega / K} r_{i 0 k}^{l 0 n}}}{r_{i 0 k}^{l 0 n}}+\sum_{m=1}^{N S_{y}}(-1)^{(m-1)} \sum_{j=1}^{4}(-1)^{((j-1) !(j+1)+1)} \frac{e^{-\sqrt{i \omega / K} r_{i j k}^{l m n}}}{r_{i j k}^{l m n}}\right]\right] \\
& T(x, y, z, \omega)=\frac{-i \pi}{2 \lambda L_{x}}\left[\sum_{m_{k}=-\infty}^{\infty}\left[H_{0}\left(k_{1 z} r_{00}^{00}\right) e^{-i k_{z}\left(\bar{z}_{0}\right)}\right]+\sum_{m=1}^{N S_{y}}(-1)^{(m-1)} \sum_{j=1}^{4}(-1)^{((j-1) !(j+1)+1)} \sum_{m_{k}=-\infty}^{\infty}\left[H_{0}\left(k_{1 z} r_{0 j}^{0 m}\right) e^{-i k_{z}\left(\bar{z}_{0}\right)}\right]\right. \\
& +\sum_{l=1}^{N S_{x}}(-1)^{(l-1)} \sum_{i=1}^{4}(-1)^{(i !+1)}\left[\sum_{m_{k}=-\infty}^{\infty}\left[H_{0}\left(k_{1 z} r_{i 0}^{l 0}\right) e^{-i k_{z}\left(\bar{z}_{0}\right)}\right]+\sum_{m=1}^{N S_{y}}(-1)^{(m-1)} \sum_{j=1}^{4}(-1)^{((j-1) !(j+1)+1)}\right. \\
& \left.\cdot \sum_{m_{k}=-\infty}^{\infty}\left[H_{0}\left(k_{1 z} r_{i j}^{l m}\right) e^{-i k_{z}\left(\bar{z}_{0}\right)}\right]\right]+\sum_{n=1}^{N S_{z}}(-1)^{(n-1)} \sum_{k=1}^{4}(-1)^{(k !+1)}\left[\sum_{m_{k}=-\infty}^{\infty}\left[H_{0}\left(k_{1 z} r_{00}^{00}\right) e^{-i k_{z}\left(\bar{z}_{n}\right)}\right]+\sum_{m=1}^{N S_{y}}(-1)^{(m-1)}\right.
\end{aligned}
$$




$$
\begin{aligned}
& \cdot \sum_{j=1}^{4}(-1)^{((j-1) !(j+1)+1)} \sum_{m_{k}=-\infty}^{\infty}\left[H_{0}\left(k_{1 z} r_{0 j}^{0 m}\right) e^{-i k_{z}\left(\bar{z}_{n}\right)}\right]+\sum_{l=1}^{N S_{x}}(-1)^{(l-1)} \\
& \left.\cdot \sum_{i=1}^{4}\left[\sum_{m_{k}=-\infty}^{\infty}\left[H_{0}\left(k_{1 z} r_{i 0}^{l 0}\right) e^{-i k_{z}\left(\bar{z}_{n}\right)}\right]+\sum_{m=1}^{N S_{y}}(-1)^{(m-1)} \sum_{j=1}^{4}(-1)^{((j-1) !(j+1)+1)} \sum_{m_{k}=-\infty}^{\infty}\left[H_{0}\left(k_{1 z} r_{i j}^{l m}\right) e^{-i k_{z}\left(\bar{z}_{n}\right)}\right]\right]\right]
\end{aligned}
$$

as the sum of $2.5 \mathrm{D}$ sources.

\section{Case 4. Consider}

$$
\begin{aligned}
& T(x, y, z, t)=\frac{1}{\rho c(4 \pi K \tau)^{1.5}}\left[e^{-\left(r_{000}^{000}\right)^{2} / 4 K \tau}\right. \\
& +\sum_{m=1}^{N S_{y}}(-1)^{(m-1)} \sum_{j=1}^{4}(-1)^{(j !+1)} e^{-\left(r_{0 j 0}^{0 m 0}\right)^{2} / 4 K \tau} \\
& +\sum_{l=1}^{N S_{x}}(-1)^{(l-1)} \sum_{i=1}^{4}(-1)^{((i-1) !(i+1)+1)}\left[e^{-\left(r_{i 00}^{l 00}\right)^{2} / 4 K \tau}\right. \\
& \left.+\sum_{m=1}^{N S_{y}}(-1)^{(m-1)} \sum_{j=1}^{4}(-1)^{(j !+1)} e^{-\left(r_{i j 0}^{l m 0}\right)^{2} / 4 K \tau}\right] \\
& +\sum_{n=1}^{N S_{z}}(-1)^{(n-1)} \sum_{k=1}^{4}(-1)^{((k-1) !(k+1)+1)}\left[e^{-\left(r_{00 k}^{00 n}\right)^{2} / 4 K \tau}\right. \\
& +\sum_{m=1}^{N S_{y}}(-1)^{(m-1)} \sum_{j=1}^{4}(-1)^{(j !+1)} e^{-\left(r_{0 j k}^{0 m n}\right)^{2} / 4 K \tau} \\
& +\sum_{l=1}^{N S_{x}}(-1)^{(l-1)} \sum_{i=1}^{4}(-1)^{((i-1) !(i+1)+1)}\left[e^{-\left(r_{i 0 k}^{l 0 n}\right)^{2} / 4 K \tau}\right. \\
& \left.\left.\left.+\sum_{m=1}^{N S_{y}}(-1)^{(m-1)} \sum_{j=1}^{4}(-1)^{(j !+1)} e^{-\left(r_{i j k}^{l m n}\right)^{2} / 4 K \tau}\right]\right]\right] \text {, } \\
& T(x, y, z, \omega)=\frac{1}{2 \lambda}\left[\frac{e^{-\sqrt{i \omega / K} r_{000}^{000}}}{r_{000}^{000}}+\sum_{m=1}^{N S_{y}}\left[(-1)^{(m-1)}\right.\right. \\
& \left.\cdot \sum_{j=1}^{4}(-1)^{(j !+1)} \frac{e^{-\sqrt{i \omega / K} r_{0 j 0}^{0 m 0}}}{r_{0 j 0}^{0 m 0}}\right]+\sum_{l=1}^{N S_{x}}(-1)^{(l-1)} \\
& \cdot \sum_{i=1}^{4}(-1)^{((i-1) !(i+1)+1)}\left[\frac{e^{-\sqrt{i \omega / K} r_{i 00}^{l 00}}}{r_{i 00}^{l 00}}+\sum_{m=1}^{N S_{y}}(-1)^{(m-1)}\right. \\
& \sum_{k=1}^{4}(-1)^{((k-1) !(k+1)+1)}\left[\frac{e^{-\sqrt{i \omega / K} r_{00 k}^{00 n}}}{r_{00 k}^{00 n}}\right. \\
& +\sum_{m=1}^{N S_{y}}\left[(-1)^{(m-1)} \sum_{j=1}^{4}(-1)^{(j !+1)} \frac{e^{-\sqrt{i \omega / K} r_{0 j k}^{0 m n}}}{r_{0 j k}^{0 m n}}\right] \\
& +\sum_{l=1}^{N S_{x}}(-1)^{(l-1)} \sum_{i=1}^{4}(-1)^{((i-1) !(i+1)+1)}\left[\frac{e^{-\sqrt{i \omega / K} r_{i 0 k}^{l 0 n}}}{r_{i 0 k}^{l 0 n}}\right. \\
& \left.\left.\left.+\sum_{m=1}^{N S_{y}}(-1)^{(m-1)} \sum_{j=1}^{4}(-1)^{(j !+1)} \frac{e^{-\sqrt{i \omega / K} r_{i j k}^{l m n}}}{r_{i j k}^{l m n}}\right]\right]\right] \\
& T(x, y, z, \omega)=\frac{-i \pi}{2 \lambda L_{x}}\left[\sum_{m_{k}=-\infty}^{\infty}\left[H_{0}\left(k_{1 z} r_{00}^{00}\right) e^{-i k_{z}\left(\bar{z}_{0}\right)}\right]\right. \\
& +\sum_{m=1}^{N S_{y}}(-1)^{(m-1)} \sum_{j=1}^{4}(-1)^{(j !+1)} \sum_{m_{k}=-\infty}^{\infty}\left[H_{0}\left(k_{1 z} r_{0 j}^{0 m}\right)\right. \\
& \left.\cdot e^{-i k_{z}\left(\bar{z}_{0}\right)}\right]+\sum_{l=1}^{N S_{x}}(-1)^{(l-1)} \sum_{i=1}^{4}(-1)^{((i-1) !(i+1)+1)} \\
& \cdot\left[\sum_{m_{k}=-\infty}^{\infty}\left[H_{0}\left(k_{1 z} r_{i 0}^{l 0}\right) e^{-i k_{z}\left(\bar{z}_{0}\right)}\right]+\sum_{m=1}^{N S_{y}}(-1)^{(m-1)}\right. \\
& \left.\cdot \sum_{j=1}^{4}(-1)^{(j !+1)} \sum_{m_{k}=-\infty}^{\infty}\left[H_{0}\left(k_{1 z} r_{i j}^{l m}\right) e^{-i k_{z}\left(\bar{z}_{0}\right)}\right]\right] \\
& +\sum_{n=1}^{N S_{z}}(-1)^{(n-1)} \sum_{k=1}^{4}(-1)^{((k-1) !(k+1)+1)} \\
& \cdot\left[\sum_{m_{k}=-\infty}^{\infty}\left[H_{0}\left(k_{1 z} r_{00}^{00}\right) e^{-i k_{z}\left(\bar{z}_{n}\right)}\right]+\sum_{m=1}^{N S_{y}}(-1)^{(m-1)}\right.
\end{aligned}
$$$$
\left.\cdot \sum_{j=1}^{4}(-1)^{(j !+1)} \frac{e^{-\sqrt{i \omega / K} r_{i j 0}^{l m 0}}}{r_{i j 0}^{l m 0}}\right]+\sum_{n=1}^{N S_{z}}(-1)^{(n-1)}
$$ 


$$
\begin{aligned}
& \cdot \sum_{j=1}^{4}(-1)^{(j !+1)} \sum_{m_{k}=-\infty}^{\infty}\left[H_{0}\left(k_{1 z} r_{0 j}^{0 m}\right) e^{-i k_{z}\left(\bar{z}_{n}\right)}\right] \\
& +\sum_{l=1}^{N S_{x}}(-1)^{(l-1)} \sum_{i=1}^{4}(-1)^{((i-1) !(i+1)+1)} \\
& \cdot\left[\sum_{m_{k}=-\infty}^{\infty}\left[H_{0}\left(k_{1 z} r_{i 0}^{l 0}\right) e^{-i k_{z}\left(\bar{z}_{n}\right)}\right]+\sum_{m=1}^{N S_{y}}(-1)^{(m-1)}\right. \\
& \left.\left.\left.\cdot \sum_{j=1}^{4}(-1)^{(j !+1)} \sum_{m_{k}=-\infty}^{\infty}\left[H_{0}\left(k_{1 z^{r}} r_{i j}^{l m}\right) e^{-i k_{z}\left(\bar{z}_{n}\right)}\right]\right]\right]\right]
\end{aligned}
$$

as the sum of $2.5 \mathrm{D}$ sources.

\section{Conclusions}

Fully analytical solutions for heat conduction for unbounded and rectangular spaces subjected to point, line, and plane sources have been presented. Two boundary conditions were assumed, namely, the Dirichlet and Neumann boundary conditions. Particular attention was given to the two-anda-half-dimensional fundamental solution or 2.5D Green's functions defined for spatially sinusoidal, harmonic line sources. The final expressions were validated by applying the equations to the problem of a Dirac delta source, for which the solutions in the time domain are known in analytical form. Excellent agreement was found between the numerical solutions given by Fourier synthesis and the exact solutions.

\section{Conflict of Interests}

The authors declare that there is no conflict of interests regarding the publication of this paper.

\section{Acknowledgments}

This work has been framed under the Initiative Energy for Sustainability of the University of Coimbra and supported by the Energy and Mobility for Sustainable Regions (EMSURE) Project (CENTRO-07-0224-FEDER-002004) and was supported in part by the POCI-01-0247-FEDER-003179 (Revi Clean Facade) Project funded by Portugal 2020 through the Operational Programme for Competitiveness Factors (COMPETE 2020).

\section{References}

[1] H. S. Carslaw and J. C. Jaeger, Conduction of Heat in Solids, Oxford University Press, 2nd edition, 1959.

[2] J. V. Beck, K. D. Cole, A. Haji-Sheikh, and B. Litkouhi, Heat Conduction using Green's Function, Hemisphere Publishing, New York, NY, USA, 2nd edition, 2010.

[3] J. V. Beck, "Green's function solution for transient heat conduction problems," International Journal of Heat and Mass Transfer, vol. 27, no. 8, pp. 1235-1244, 1984.
[4] A. E. Joanni and E. Kausel, "Heat diffusion in layered media via the thin-layer method," International Journal for Numerical Methods in Engineering, vol. 78, no. 6, pp. 692-712, 2009.

[5] A. Tadeu, I. Simões, N. Simões, and J. Prata, "Simulation of dynamic linear thermal bridges using a boundary element method model in the frequency domain," Energy and Buildings, vol. 43, no. 12, pp. 3685-3695, 2011.

[6] A. Tadeu and N. Simões, "Three-dimensional fundamental solutions for transient heat transfer by conduction in an unbounded medium, half-space, slab and layered media," Engineering Analysis with Boundary Elements, vol. 30, no. 5, pp. 338349, 2006.

[7] A. Tadeu, I. Simões, J. António, and L. Sousa, "Simulation of the 3D sound pressure level inside closed absorbing acoustic rooms bounded by non-parallel floor and ceiling surfaces, and parallel sidewalls," Acta Acustica united with Acustica, vol. 98, no. 6, pp. 894-906, 2012.

[8] J.-T. Chen, H.-C. Shieh, J.-J. Tsai, and J.-W. Lee, "A study on the method of fundamental solutions using an image concept," Applied Mathematical Modelling, vol. 34, no. 12, pp. 4253-4266, 2010.

[9] J.-T. Chen, H.-C. Shieh, Y.-T. Lee, and J.-W. Lee, "Image solutions for boundary value problems without sources," Applied Mathematics and Computation, vol. 216, no. 5, pp. 1453-1468, 2010.

[10] J.-T. Chen, Y.-T. Lee, S.-R. Yu, and S.-C. Shieh, "Equivalence between the Trefftz method and the method of fundamental solution for the annular Green's function using the addition theorem and image concept," Engineering Analysis with Boundary Elements, vol. 33, no. 5, pp. 678-688, 2009. 


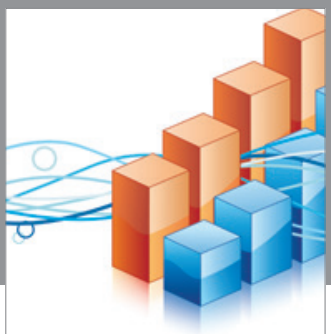

Advances in

Operations Research

vatem alat4

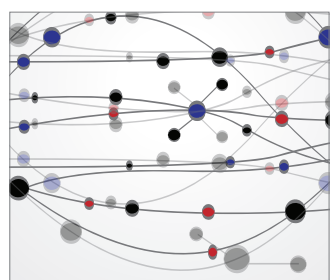

\section{The Scientific} World Journal
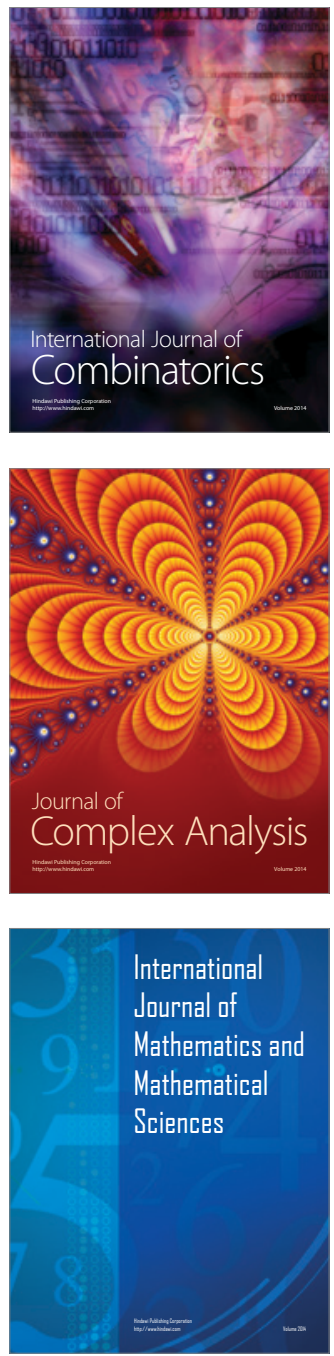
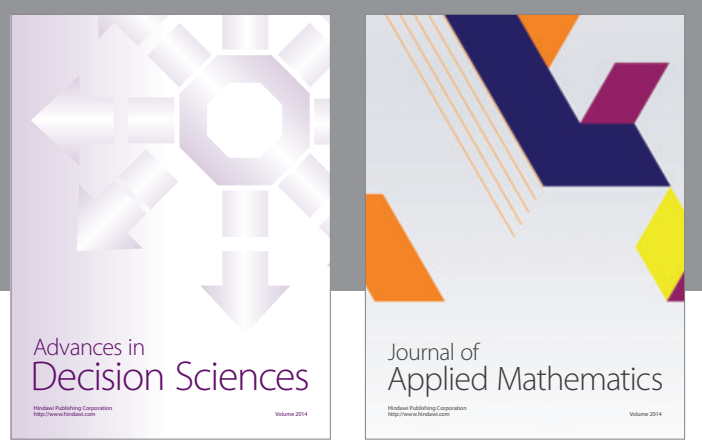

Algebra

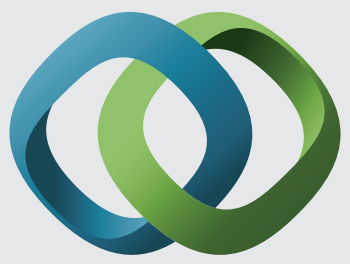

\section{Hindawi}

Submit your manuscripts at

http://www.hindawi.com
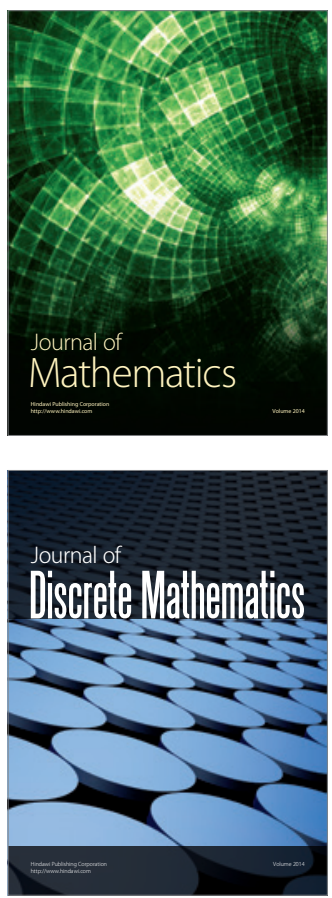

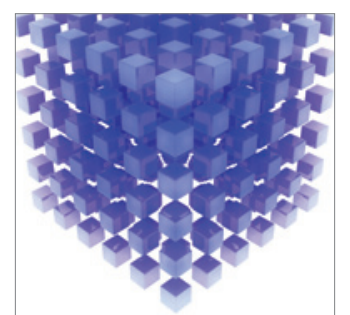

Mathematical Problems in Engineering
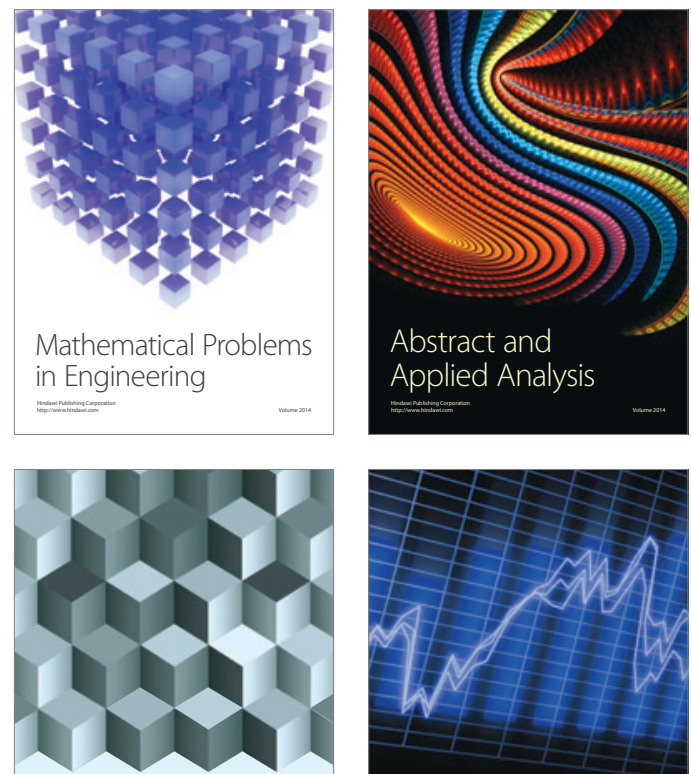

Journal of

Function Spaces

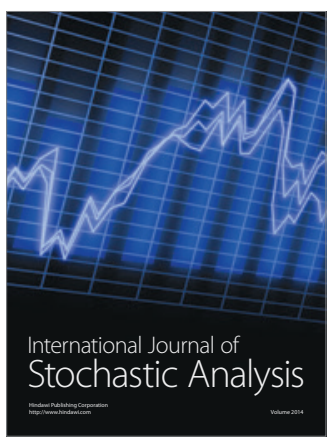

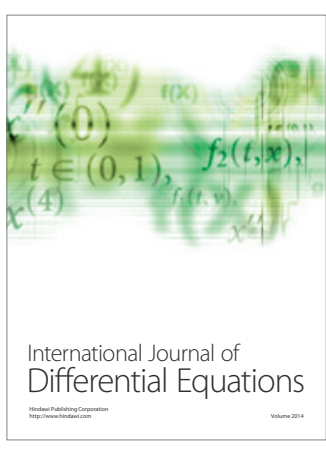
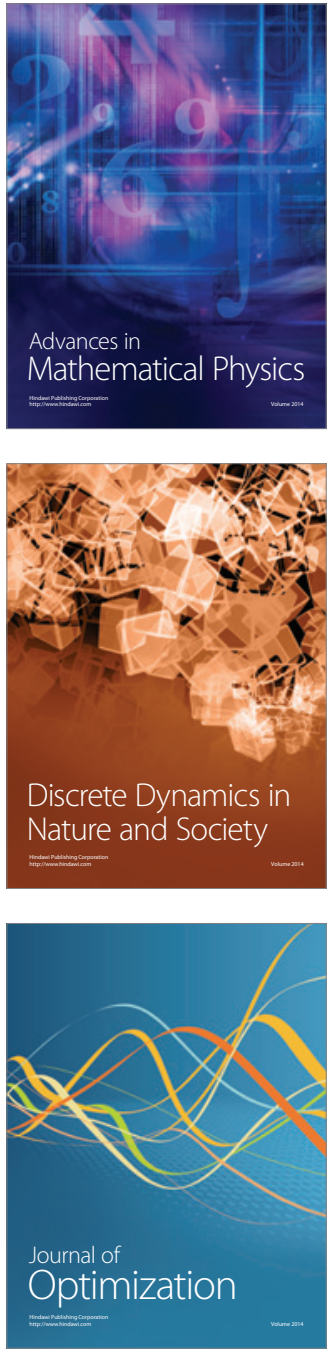